\title{
The Velocity Dispersion Function of Very Massive Galaxy Clusters: Abell 2029 and Coma
}

Jubee Sohn

Margaret J. Geller

H. Jabran Zahid

Daniel G. Fabricant

Antonaldo Diaferio

See next page for additional authors

Follow this and additional works at: https://cedar.wwu.edu/physicsastronomy_facpubs

Part of the Astrophysics and Astronomy Commons

\section{Recommended Citation}

Sohn, Jubee; Geller, Margaret J.; Zahid, H. Jabran; Fabricant, Daniel G.; Diaferio, Antonaldo; and Rines, Kenneth J., "The Velocity Dispersion Function of Very Massive Galaxy Clusters: Abell 2029 and Coma" (2017). Physics \& Astronomy. 33.

https://cedar.wwu.edu/physicsastronomy_facpubs/33 
Authors

Jubee Sohn, Margaret J. Geller, H. Jabran Zahid, Daniel G. Fabricant, Antonaldo Diaferio, and Kenneth J. Rines 


\title{
The Velocity Dispersion Function of Very Massive Galaxy Clusters: Abell 2029 and Coma
}

\author{
Jubee Sohn ${ }^{1}$, Margaret J. Geller ${ }^{1}$, H. Jabran Zahid ${ }^{1}$, Daniel G. Fabricant ${ }^{1}$, Antonaldo Diaferio ${ }^{2,3}$, and Kenneth J. Rines ${ }^{4}$ \\ ${ }^{1}$ Smithsonian Astrophysical Observatory, 60 Garden Street, Cambridge, MA 02138, USA \\ ${ }^{2}$ Università di Torino, Dipartimento di Fisica, Torino, Italy \\ ${ }^{3}$ Istituto Nazionale di Fisica Nucleare (INFN), Sezione di Torino, Torino, Italy \\ ${ }^{4}$ Department of Physics \& Astronomy, Western Washington University, Bellingham, WA 98225, USA \\ Received 2016 December 19; revised 2017 February 24; accepted 2017 February 24; published 2017 March 29
}

\begin{abstract}
Based on an extensive redshift survey for galaxy clusters Abell 2029 and Coma, we measure the luminosity functions (LFs) and stellar mass functions (SMFs) for the entire cluster member galaxies. Most importantly, we measure the velocity dispersion functions (VDFs) for quiescent members. The MMT/Hectospec redshift survey for galaxies in A2029 identifies 982 spectroscopic members; for 838 members, we derive the central velocity dispersion from the spectroscopy. Coma is the only other cluster surveyed as densely. The LFs, SMFs, and VDFs for A2029 and Coma are essentially identical. The SMFs of the clusters are consistent with simulations. The A2029 and Coma VDFs for quiescent galaxies have a significantly steeper slope than those of field galaxies for velocity dispersion $\lesssim 100 \mathrm{~km} \mathrm{~s}^{-1}$. The cluster VDFs also exceed the field at velocity dispersion $\gtrsim 250 \mathrm{~km} \mathrm{~s}^{-1}$. The differences between cluster and field VDFs are potentially important tests of simulations and of the formation of structure in the universe.
\end{abstract}

Key words: galaxies: clusters: individual (Coma, Abell 2029) - galaxies: distances and redshifts - galaxies: fundamental parameters - galaxies: luminosity function, mass function

Supporting material: machine-readable table

\section{Introduction}

Statistical analyses of galaxy properties provide fundamental tests of structure formation models. In the standard $\Lambda \mathrm{CDM}$ model, dark matter (DM) halos govern structure formation. Once DM halos form, baryonic physics plays a role in forming galaxies within the DM halo. Because all observable quantities are related to baryonic matter, finding connections between baryonic matter and the DM halo is a central goal. In particular, identifying the best DM halo tracers among observables is a key issue.

The luminosity of individual galaxies is a fundamental observable. The luminosity function (LF) has conventionally been used to test structure formation models (e.g., Klypin et al. 1999; Vale \& Ostriker 2004, 2006; Yang et al. 2008). However, connecting the LF to the mass distribution of DM halos is non-trivial.

Stellar mass has recently come into the spotlight as a better tracer of halo masses (e.g., Behroozi et al. 2010; More et al. 2011; Li et al. 2013; Tinker et al. 2016). Like luminosity, stellar mass is also governed by baryonic physics, but it appears to be more closely related to the DM properties (More et al. 2011; Li et al. 2013). Thus, the stellar mass function (SMF) is a basis for matching galaxies to DM subhalos (e.g., the abundance matching technique; Kravtsov et al. 2004; Conroy et al. 2006; Guo et al. 2010) when comparing observations with cosmological simulations. The SMF may, in principle, depend on galaxy morphology, color, redshift, and environment (e.g., Calvi et al. 2013; Vulcani et al. 2013). The shape of the SMF depends on the galaxy morphology and color. Variations in the SMF for the total galaxy population with redshift and environment are hard to detect because the expected differences are apparent only for low stellar masses where nearly all galaxies are star-forming.
Central velocity dispersion may be a more fundamental tracer of the DM halo (Wake et al. 2012; van Uitert et al. 2013; Bogdán \& Goulding 2015; Zahid et al. 2016). The central velocity dispersion reflects the stellar kinematics governed by the central gravitational potential well. Moreover, the velocity dispersion is a direct dynamical measurement, whereas luminosity and stellar mass measurements suffer from various systematic issues and model dependence (e.g., Conroy et al. 2009).

Taking advantage of huge galaxy surveys, many studies analyze statistical properties of galaxies in the general field, including LFs (e.g., Blanton et al. 2001; Loveday et al. 2012; McNaught-Roberts et al. 2014), SMFs (e.g., Vulcani et al. 2011, 2013; Mortlock et al. 2015), and velocity dispersion functions (VDFs, e.g., Sheth et al. 2003; Choi et al. 2007; Chae 2010; Montero-Dorta et al. 2016). Using these large samples, the effects of galaxy morphology, environment, and redshift have also been investigated. However, computation of the LFs, SMFs, and VDFs for an identical sample of galaxies is rare. Bernardi et al. (2010) explore the LF, SMF, and VDF simultaneously based on the Sloan Digital Sky Survey (SDSS) data release 6 (DR6). They sample all environments, covering a wide redshift range.

Galaxy clusters offer another test bed for the statistical study of galaxy properties. Because galaxies in a cluster are essentially at a fixed redshift and share a common dense environment, samples of cluster galaxies complement samples from general surveys. Statistical studies of spectroscopically confirmed cluster members control for some observational biases. Nonetheless, there are few studies that explore the LF (Rines \& Geller 2008; Agulli et al. 2014, 2016; Lee et al. 2016) or the SMF (Ferrarese et al. 2016) of spectroscopically identified members. In contrast, there are many studies based on photometrically determined membership (e.g., Barkhouse 
Table 1

Basic Properties of the Coma and A2029

\begin{tabular}{|c|c|c|c|c|c|c|c|c|}
\hline Name & $\begin{array}{l}\text { R.A. }^{\mathrm{a}} \\
(\mathrm{J} 2000)\end{array}$ & $\begin{array}{c}\text { Decl. }^{\mathrm{a}} \\
(\mathrm{J} 2000)\end{array}$ & $z^{\mathrm{a}}$ & $\begin{array}{c}\sigma_{c l}^{\mathrm{b}} \\
\left(\mathrm{km} \mathrm{s}^{-1}\right)\end{array}$ & $\begin{array}{l}R_{200}{ }^{\mathrm{a}} \\
(\mathrm{Mpc})\end{array}$ & $\begin{array}{r}M_{200}{ }^{\mathrm{a}} \\
\left(10^{15} M_{\odot}\right)\end{array}$ & $N_{\text {mem }}$ & $\begin{array}{c}N_{\text {mem }} \\
\left(R_{c l}<R_{200}\right)\end{array}$ \\
\hline Coma & $13: 00: 23.8$ & $+27: 56: 39$ & 0.0235 & $947 \pm 31$ & $2.23_{-0.09}^{+0.08}$ & $1.29_{-0.15}^{+0.15}$ & 1224 & 856 \\
\hline A2029 & $15: 10: 57.2$ & $+05: 45: 16$ & 0.0784 & $973 \pm 31$ & $1.97_{-0.21}^{+0.20}$ & $0.94_{-0.27}^{+0.30}$ & 982 & 518 \\
\hline
\end{tabular}

Notes.

a Estimated from the caustic technique.

${ }^{\mathrm{b}}$ Calculated using spectroscopically identified members within $R_{200}$.

et al. 2007; Moretti et al. 2015; Lan et al. 2016; Lee \& Jang 2016).

Measurements of the VDF for an individual galaxy cluster are rare. Only Munari et al. (2016) examine the VDF in a galaxy cluster, A2142. They use SDSS spectra to measure the velocity dispersions. Then, they convert the velocity dispersions into circular velocities to construct a circular velocity function. Finally, they compare the circular velocity function of A2142 to the circular velocity functions of subhalos in a set of numerical simulations. They take this approach because direct calculation of the velocity dispersion from simulations to mimic the observations are not yet available. They suggest that current numerical simulations underestimate the number of massive $\left(>200 \mathrm{~km} \mathrm{~s}^{-1}\right)$ subhalos.

Here, we investigate the LFs, SMFs, and VDFs for Coma and A2029, two very massive clusters $\left(>4 \times 10^{14} M_{\odot}\right.$, Rines et al. 2016). Our analysis is based on an essentially complete sample of $\sim 1000$ spectroscopically identified members in each system. We first examine the well-studied LFs and SMFs and use those functions to guide our development of the cluster VDFs. Comparisons among these observables provide a basis for modeling the connection between DM halos, which are possibly traced by the central velocity dispersion (Bogdán \& Goulding 2015; Zahid et al. 2016). We describe the data in Section 2 and the member selection in Section 3. We investigate the LFs, SMFs, and VDFs in Section 4. We discuss the results in Section 5 and summarize in Section 6. We adopt the standard cosmology of $H_{0}=70 \mathrm{~km} \mathrm{~s}^{-1} \mathrm{Mpc}^{-1}$, $\Omega_{m}=0.3$, and $\Omega_{\Lambda}=0.7$ throughout.

\section{Observations of A2029 and Coma}

Abell $2029(z=0.078)$ and Coma $(z=0.023)$ are two of the most massive galaxy clusters in the nearby universe. Thus, they are ideal for studying the LFs, SMFs, and VDFs for large samples of cluster members. Table 1 summarizes the basic properties of the two clusters.

\subsection{A2029}

A2029 is a massive cluster with a dominant cD galaxy (IC $1101)$. The $\mathrm{cD}$ has an extremely large halo ( $600 \mathrm{kpc}$; Uson et al. 1991). The velocity dispersion of the $\mathrm{cD}$ increases with cluster-centric distance (Fisher et al. 1995). Although A2029 appears relaxed in the optical, X-ray observations reveal an extended X-ray sloshing spiral structure indicative of complex internal dynamics in the intracluster medium (Clarke et al. 2004; Walker et al. 2012; Paterno-Mahler et al. 2013). To study the statistical properties of A2029 member galaxies, we carry out a redshift survey using Hectospec (Fabricant et al. 2008), a wide-field spectrograph on the $6.5 \mathrm{~m} \mathrm{MMT.}$

\subsubsection{Photometric Data}

We use photometric data from SDSS DR12 (Alam et al. 2015) as the basis for the redshift survey. We first select extended sources brighter than $r=22$ mag within a projected radius $R_{c l}<100^{\prime}$ from the cluster center (R.A., Decl.: 227.73813, 5.7544). Unfortunately, the SDSS DR12 photometric catalog is incomplete in the north-eastern part of A2029 where there is a small patch of missing photometry. To correct for the incompleteness of the SDSS DR12 photometry, we compiled SDSS DR7 photometry for the missing region.

We use composite model magnitudes as the SDSS web pages recommend. The composite model magnitude is a linear combination of de Vaucouleurs and exponential magnitudes, yielding an approximately Petrosian magnitude. We apply the extinction correction for each band provided in the SDSS photometric catalog. Hereafter, all magnitudes denote extinction-corrected composite model magnitudes.

Bernardi et al. (2013) suggest that photometry from the SDSS DR7 pipeline underestimates galaxy luminosities. The cModel apparent magnitude is more of an overestimate for more luminous objects. Thus stellar mass estimates derived from cModel magnitudes may be underestimates; the underestimates appear to be more serious for more massive galaxies. The analysis of Bernardi et al. (2013) applies to a large sample of field galaxies with redshift $z \leqslant 0.06$. Analysis of Coma and A2029 present even more complex issues including measurement of intracluster light and the treatment of crowded fields. The two clusters are also at significantly different redshifts and A2029 is significantly beyond the redshift limit of the analysis of Bernardi et al. (2013). We thus take the SDSS DR12 photometry at face value, but caution that deeper data in better seeing might modify the results.

Figure 1 shows the color-magnitude diagram for the A2029 region. We plot spectroscopic targets (see Section 2.1.2) and spectroscopically identified members (see Section 3). Following Rines et al. (2013), we identify the red sequence of A2029 by assuming a slope of -0.04 in the $g-r$ versus $r$ colormagnitude diagram. We consider objects within \pm 0.1 of the relation as potential red-sequence members. Hereafter, we refer to the galaxies that are bluer/redder than the red sequence as blue and very red galaxies.

\subsubsection{Spectroscopy}

Spectroscopic redshifts are the best way to determine cluster membership. Contamination by foreground and background objects is significantly reduced relative to samples based on photometric redshifts (e.g., Geller et al. 2014; Hwang et al. 2014). Applying the caustic technique (Diaferio \& Geller 1997; Diaferio 1999; Serra \& Diaferio 2013) to the spectroscopic 


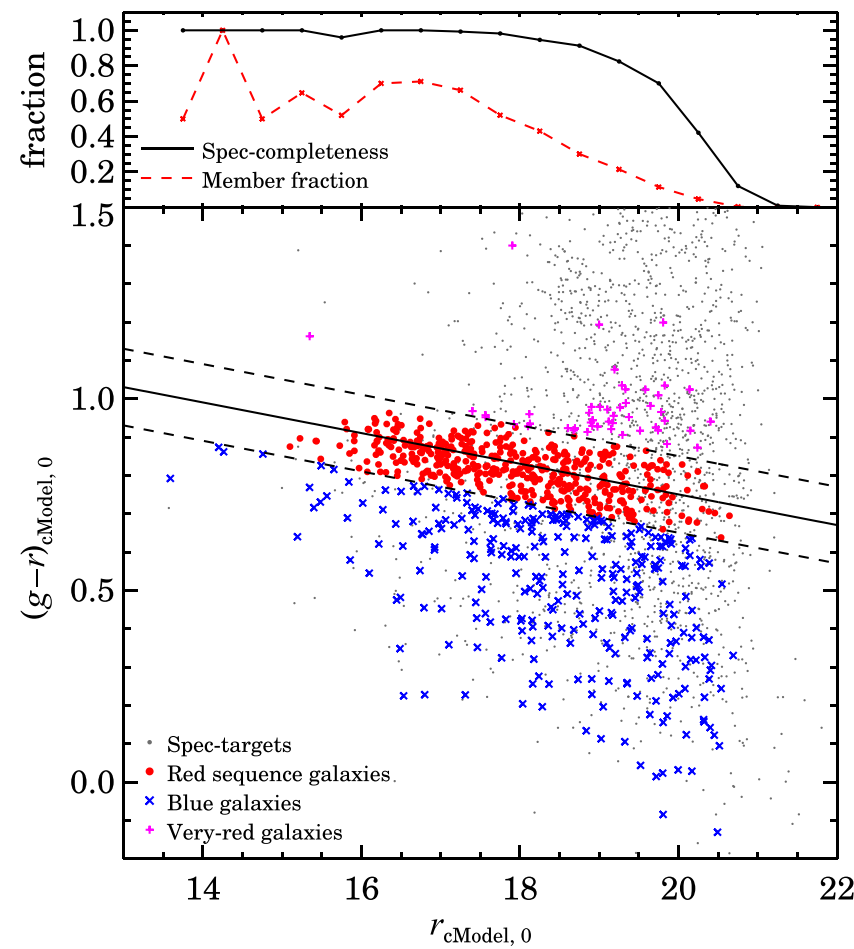

Figure 1. Color-magnitude ( $g-r$ vs. $r$ ) diagram for A2029. Filled circles, crosses, and pluses represent red-sequence, blue, and very red member galaxies, respectively. Gray dots show A2029 survey field galaxies with redshifts. The solid line shows the red sequence of A2029 and the dashed lines show the boundaries of the red sequence following Rines \& Geller (2008).

sample (see Section 3) identifies cluster members; the completeness of membership determination from the caustic technique is $95 \%$ within $3 R_{200}$ based on numerical simulations (Serra \& Diaferio 2013).

For bright A2029 galaxies we compiled redshifts from the SDSS spectroscopic survey. SDSS spectra are acquired through $3^{\prime \prime}$ fibers for galaxies brighter than $r=17.77$. There are 2807 SDSS redshifts in the A2029 field $\left(R_{c l}<100^{\prime}\right)$. The typical measurement error for SDSS redshifts is $13 \mathrm{~km} \mathrm{~s}^{-1}$.

We also collected redshifts from the literature. We compiled 40 redshifts from the NASA/IPAC Extragalactic Database (NED) and one redshift from the $1.5 \mathrm{~m}$ telescope on Mt. Hopkins (Sohn et al. 2015). Tyler et al. (2013) previously conducted a redshift survey for A2029 using the MMT/ Hectospec with the 270 line $\mathrm{mm}^{-1}$ grating. They obtained 1164 spectra and identified cluster members to investigate the infrared properties of A2029 galaxies. We collected these spectra from the MMT archive. ${ }^{5}$ Because SDSS DR12 revisited the A2029 area after the Hectospec survey, there are 296 objects with both Hectospec and SDSS spectroscopy in this subsample. We use these spectra for relative calibration of the velocity dispersions derived from the two samples.

We carried out our redshift survey of A2029 using MMT/ Hectospec between April and June 2016. The Hectospec instrument mounted on the MMT $6.5 \mathrm{~m}$ telescope (Fabricant et al. 2005) is a multi-object fiber-fed spectrograph with 300 fibers covering an $\sim 1 \mathrm{deg}^{2}$ field of view, i.e., $R_{\text {Hecto }}=30^{\prime}$. We used the 270 line $\mathrm{mm}^{-1}$ grating and the resulting spectra cover the wavelength range $\lambda=3700-9150 \AA$ with a resolution of

\footnotetext{
5 http://oirsa.cfa.harvard.edu/archive/search/
}

$6.2 \AA$. For each Hectospec field, we obtained three sequential exposures of $1200 \mathrm{~s}$ each. Tyler et al. (2013) used the same integration times for their Hectospec observations; thus we include their spectra and measurements without any correction.

We select galaxies brighter than $r=21.3$ from the SDSS photometric galaxy catalog as Hectospec targets. We exclude galaxies with fiber magnitude $r_{\text {fib }}>22$ from the target list; these galaxies have a surface brightness too low to yield a reliable Hectospec redshift with our integration time. We apply no color selection to the target list.

We reduce the data with the HSRED v2.0 package, a Hectospec pipeline developed by Richard Cool. We measure the redshifts using RVSAO (Kurtz \& Mink 1998), which crosscorrelates the spectra with a set of templates constructed for this purpose (Fabricant et al. 2005). We visually inspect all spectra and divided the cross-correlation results into three groups: "Q" for high-quality redshift, "?" for marginal cases, "X" for poor fits. We use only "Q" type spectra. In total, we obtain 1597 high-quality redshifts with a median measurement error of $29 \mathrm{~km} \mathrm{~s}^{-1}$.

Figure 2 shows the spectroscopic completeness of the A2029 field as a function of radius and $r$-band magnitude. The spectroscopic survey is remarkably complete for $r<19.0$ and $R_{c l}<30^{\prime}$. The spectroscopic completeness drops rapidly for fainter magnitudes and for radii larger than $R_{\text {Hecto }} \sim 30^{\prime}$.

\subsubsection{Stellar Masses}

To consistently compare our results with previous studies, we measure stellar masses using the Le PHARE ${ }^{6}$ code developed by Arnout \& Ilbert (Arnouts et al. 1999; Ilbert et al. 2006). We fit the stellar energy distribution (SED) measured from SDSS broadband photometry with stellar population synthesis (SPS) models to determine the mass-tolight ratio. We use the mass-to-light ratio to convert the observed luminosity into an estimate of stellar mass. We adopt the SPS models of Bruzual \& Charlot (2003) and a Chabrier (2003) IMF. The SPS models have two metallicities. We generate synthetic SEDs by varying the star formation history (SFH), extinction, and stellar population age. We adopt exponentially declining SFHs with e-folding times ranging between 0 and $30 \mathrm{Gyr}$, the Calzetti et al. (2000) extinction law with $E(B-V)$ ranging between 0 and 0.6 , and stellar population ages between 0.01 and $13 \mathrm{Gyr}$. We generate a probability distribution function (PDF) for the stellar masses by $\chi^{2}$-fitting the synthetic SEDs to the observed photometry. We adopt the median of the PDF as our estimate of the stellar mass.

The stellar mass estimation using the Le PHARE model has $\sim 0.1-0.2$ dex offsets relative to many other approaches (Swindle et al. 2011; Zahid et al. 2014; Mobasher et al. 2015). This systematic offset reduces the apparent number of objects with stellar mass $>10^{11} M_{\odot}$. Stellar masses calculated from broadband photometry carry absolute uncertainties of $\sim 0.3$ dex (Conroy et al. 2009).

\subsubsection{Velocity Dispersions}

For galaxies with SDSS spectroscopy, we take velocity dispersions from the Portsmouth reduction (Thomas et al. 2013), because they are consistent with the velocity dispersion measured from Hectospec (Fabricant et al. 2013;

\footnotetext{
6 http://www.cfht.hawaii.edu/ãrnouts/LEPHARE/lephare.html
} 


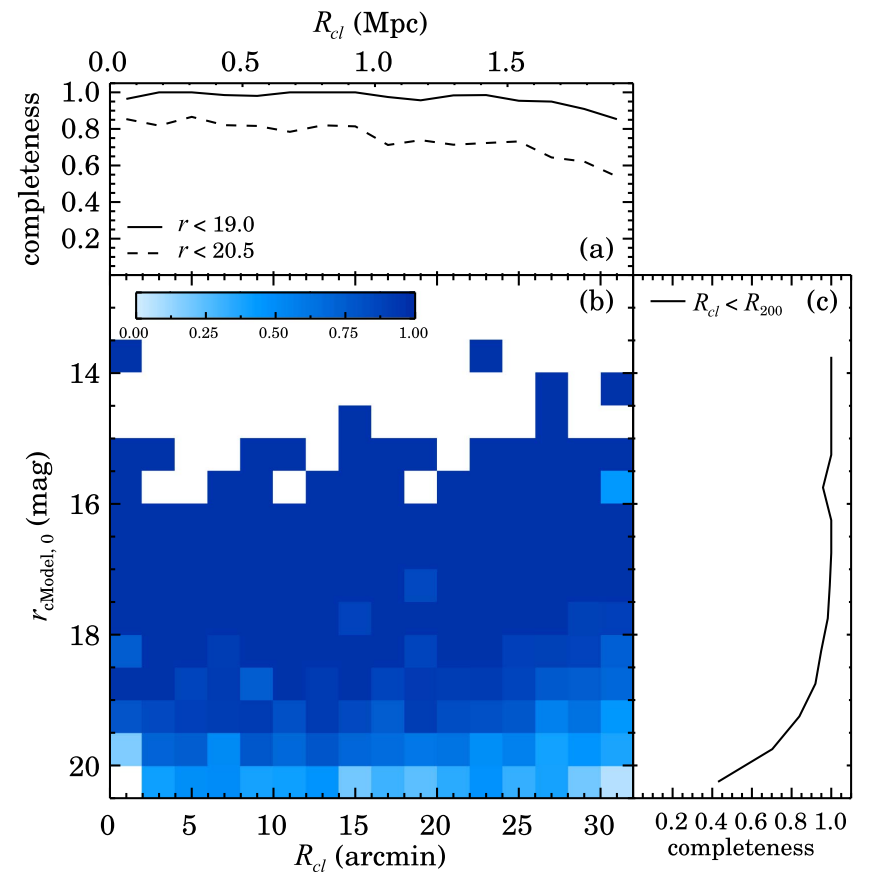

Figure 2. Two-dimensional fractional completeness of the A2029 redshift survey for $R_{c l}<R_{200}$ and $r<20.5$. Solid and dashed lines in the upper panels show the completeness to $r=19.0$ and $r=20.5$, respectively. In the right panel, the solid line displays the completeness as a function of magnitude within $R_{c l}<R_{200}$.

Zahid et al. 2016). Thomas et al. (2013) measure the velocity dispersion using the Penalized Pixel-Fitting (pPXF) code (Cappellari \& Emsellem 2004) and the stellar population templates from Maraston \& Strömbäck (2011). These templates are based on the MILES stellar library (Sánchez-Blázquez et al. 2006). They convert the templates to the SDSS resolution and derive the best-fit velocity dispersion. The median uncertainty of velocity dispersion measurement from SDSS spectroscopy is $7 \mathrm{~km} \mathrm{~s}^{-1}$.

We measure velocity dispersions for all of the galaxies with Hectospec spectroscopy, (our targets and the targets of Tyler et al. 2013) using the University of Lyon Spectroscopic analysis Software (ULySS, Koleva et al. 2009). ULySS compares the Hectospec spectra with stellar population templates calculated with the PEGASE-HR code (Le Borgne et al. 2004) and the MILES stellar library. The templates are convolved to the Hectospec resolution at varying velocity dispersions. They are parameterized by age and metallicity. ULySS determines the best-fit age, metallicity, and velocity dispersion from a chi-square fit of the convolved templates to each spectrum. We limit the fit to the rest-frame spectral range 4100-5500 A. This spectral range minimizes the uncertainty in the velocity dispersion (Fabricant et al. 2013). The median uncertainty of the velocity dispersion measurement is $\sim 17 \mathrm{~km} \mathrm{~s}^{-1}$.

Because SDSS and Hectospec spectroscopy are obtained through fibers of $3^{\prime \prime}$ and 1 ." 5 apertures, respectively, an aperture correction is necessary (Zahid et al. 2016):

$$
\left(\sigma_{\text {SDSS }} / \sigma_{\text {Hecto }}\right)=\left(R_{\text {SDSS }} / R_{\text {Hecto }}\right)^{\beta} \text {. }
$$

Following Zahid et al. (2016), we determine the aperture correction using the 169 objects with both SDSS and Hectospec velocity dispersions in the range $100<\sigma<450 \mathrm{~km} \mathrm{~s}^{-1}$ and with a velocity dispersion uncertainty smaller than $100 \mathrm{~km} \mathrm{~s}^{-1}$. The coefficient for the aperture correction is $\beta=-0.054 \pm 0.005$, consistent with previous determinations: $\beta=-0.066 \pm 0.035$ from Cappellari \& Emsellem (2004) and $\beta=-0.046 \pm 0.013$ from Zahid et al. (2016). This correction is small; our results are not sensitive to the value of $\beta$.

Below we compare the VDFs of A2029 and Coma. These two target clusters are located at different redshifts. Thus the velocity dispersions through the fiber apertures trace different portions of the target galaxies. Therefore, we correct the velocity dispersions to a fiducial physical aperture of $3 \mathrm{kpc}$ following Zahid et al. (2016). We employ Equation (1) again for this process. Hereafter, all central velocity dispersion, $\sigma$, represents the value within a $3 \mathrm{kpc}$ (rest-frame) aperture.

\subsection{5. $D_{n} 4000$}

The $D_{n} 4000$ index is defined as the ratio of flux in two spectral windows adjacent to the $4000 \AA$ break (Balogh et al. 1999). We calculate the index by taking the flux (measured per unit frequency) in the interval 4000-4100 $\AA$ relative to the flux in the interval $3850-3950 \AA$. The $D_{n} 4000$ index is sensitive to the stellar population age (Kauffmann et al. 2003; Geller et al. 2014). The $D_{n} 4000$ index also has some metallicity dependence (Kauffmann et al. 2003; Woods et al. 2010). Because the distribution of $D_{n} 4000$ is bimodal, $D_{n} 4000$ can be used to separate star-forming and quiescent galaxies spectroscopically (Mignoli et al. 2005; Vergani et al. 2008; Woods et al. 2010).

For SDSS galaxies, we adopt the $D_{n} 4000$ value from the MPA/JHU catalog. ${ }^{7}$ For BOSS and Hectospec data, we calculate $D_{n} 4000$ directly from the spectra. Fabricant et al. (2008) show that $D_{n} 4000$ measured from Hectospec and SDSS spectroscopy are consistent to within $\sim 5 \%$. This level of consistency is sufficient for our application.

\subsection{Coma}

We also use SDSS DR12 photometry for Coma galaxies. Because of the proximity of Coma, we construct the galaxy sample from a larger area, $R_{c l}<300^{\prime}$, corresponding to $100^{\prime}$ for A2029. As in the A2029 sample, we use extinctioncorrected composite model magnitudes.

Figure 3 displays the color-magnitude diagram for Coma. We determine the red-sequence using the same method applied to A2029. The red-sequence appears bluer than for A2029 because Coma is three times closer. The red-sequence of the two clusters appears in the same color range if we applying appropriate $K$-correction.

Most redshifts for Coma come from SDSS DR12 and BOSS. Although SDSS surveys a huge field, there is some residual incompleteness in dense regions due to fiber collisions (Strauss et al. 2002; Park \& Hwang 2009; Shen et al. 2016). We thus compile missing redshifts from the literature, mainly from NED (for more details, see Hwang et al. 2010). In total, we compile 22410 redshifts within $R_{c l}<300^{\prime}$. Figure 4 shows the spectroscopic completeness of Coma. The spectroscopy for the Coma field is highly complete to $r \sim 17.77$, the spectroscopic limit of the SDSS.

Using the same technique as for A2029, we measure the stellar masses of Coma galaxies. Similar to the A2029 galaxies

http://www.mpa-garching.mpg.de/SDSS/DR7/ 


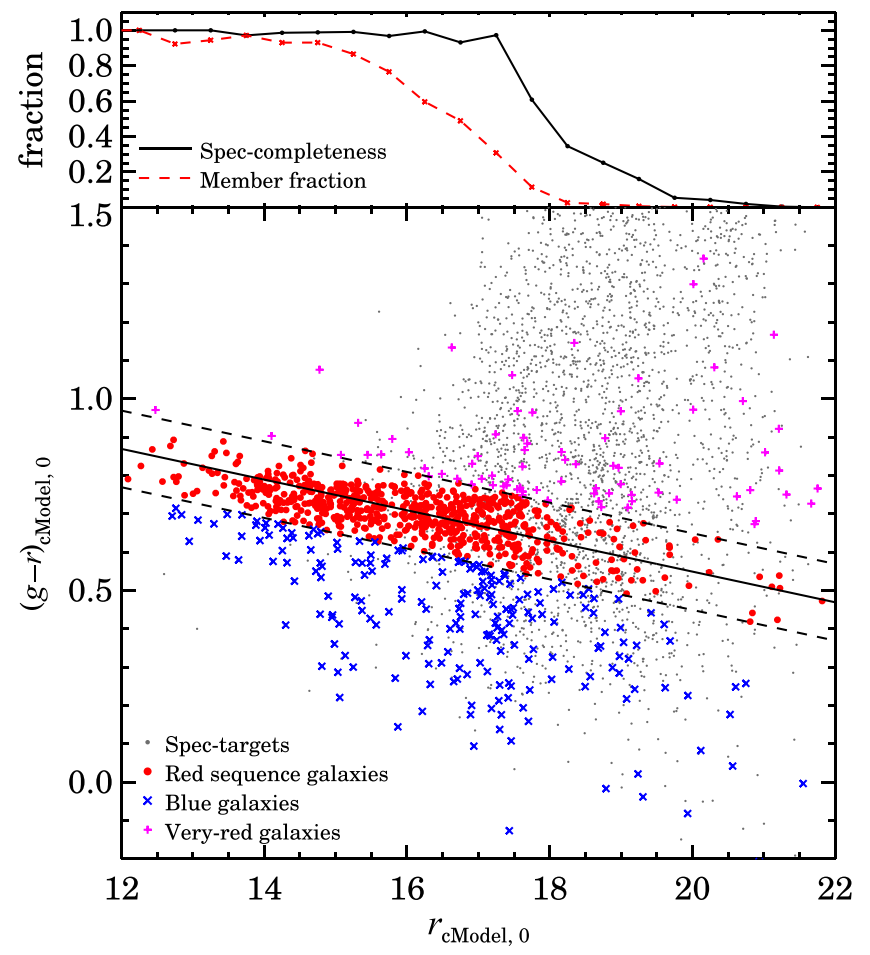

Figure 3. Same as Figure 1, but for Coma.

with SDSS spectroscopy, we calculate fiducial central velocity dispersions within $3 \mathrm{kpc}$ by applying Equation (1) to the measurement of Thomas et al. (2013). The median uncertainty of the velocity dispersions for Coma members is $\sim 4 \mathrm{~km} \mathrm{~s}^{-1}$. $D_{n} 4000$ s of Coma galaxies are from the MPA/JHU catalog.

\section{Cluster Membership}

We analyze the distribution of galaxies in phase space to identify cluster members. We use the caustic technique (Diaferio \& Geller 1997; Diaferio 1999; Serra \& Diaferio 2013) to analyze the data. Simulations by Serra \& Diaferio (2013) show that the caustic technique recovers $95 \%$ of the clusters members within $3 R_{200}$ for mock catalogs with $\sim 1000$ galaxies in the field of view and $\sim 180$ members per cluster; these objects all appear within the caustics. The interloper contamination from non-member galaxies that appear within the caustics is $\sim 2 \%$ at $R_{200}$ and $\sim 8 \%$ at $3 R_{200}$. The performance of the caustic technique may be even better for very rich clusters like Coma and A2029 that contain $~ 1000$ members.

\section{1. $A 2029$}

Figure 5 shows rest-frame cluster-centric velocity versus projected cluster-centric distance, the $R-v$ diagram, for A2029. There is a clear concentration around the center of the cluster. The non-parametric caustic technique identifies the sharp boundaries of the cluster in Figure 5. We identify objects within the caustics as cluster members. Within the caustic, there are 982 members with a mean redshift $z=0.078$. Figure 1 shows that $60 \%$ of cluster members are on the red sequence; $34 \%$ and $6 \%$ of the galaxies are blue and very red, respectively. Not all objects with red-sequence colors are cluster members; $41 \%$ of the spectroscopic targets on the red sequence are non-members. Table 2 lists the redshift and $\sigma$ for each A2029 member galaxy.

Diaferio \& Geller (1997) and Diaferio (1999) identify the caustics with the escape velocity from the cluster. This identification in turn provides the mass profile as a function of projected distance from the cluster center (Serra et al. 2011).

From the caustics in Figure 5, we compute the characteristic mass $M_{200}$ and radius $R_{200}$ where the mean density is 200 times the critical density of the universe. Table 1 lists the measured values for A2029; $R_{200}=1.97_{-0.21}^{+0.20} \mathrm{Mpc}$ and $M_{200}=$ $0.94_{-0.27}^{+0.30} \times 10^{15} M_{\odot}$. The derived $R_{200}$ and $M_{200}$ are consistent with estimates from X-ray observations; $R_{200}=1.92_{-0.13}^{+0.11} \mathrm{Mpc}$ and $M_{200}=8.0_{-1.5}^{+1.5} \times 10^{14} M_{\odot} \quad$ (Walker et al. 2012). The velocity dispersion within $R_{200}, \sigma_{c l}=973 \pm 32 \mathrm{~km} \mathrm{~s}^{-1}$, is also consistent with $\sigma_{c l}=954_{-61}^{+76} \mathrm{~km} \mathrm{~s}^{-1}$ from Rines et al. (2016).

\subsection{Coma}

Figure 6 shows the $R-v$ diagram for Coma. The caustics of Coma look smoother than those of A2029. Coma galaxies extend over a very wide region even beyond $R_{c l}=3 \mathrm{deg}$. We restrict our plot to $R_{c l} \sim 3 \mathrm{deg}$. This range is still much larger than the characteristic scale of Coma (e.g., $R_{200} \sim 1$.8 $\sim 2.1 \mathrm{Mpc}$, Geller et al. 1999). Within the caustic, we identify 1251 members with a mean redshift $z=0.0231$. The fraction of each population in Coma (Figure 3) is similar to that of A2029; 66\% are red-sequence galaxies, $25 \%$ and $9 \%$ are blue and very red members, respectively.

We measure $R_{200}$ and $M_{200}$ for Coma (Table 1) based on the caustics. Coma is slightly larger and more massive than A2029 with $R_{200}=2.23_{-0.08}^{+0.08} \mathrm{Mpc}$ and $M_{200}=1.29_{-0.15}^{+0.15} \times 10^{15} M_{\odot}$. The $R_{200}$ is consistent with the previous caustic measurement (Geller et al. 1999). The caustic mass is consistent with the weak lensing mass measurement, $M_{200}=8.9_{-2.0}^{+3.6} \times 10^{14} M_{\odot}$ (Okabe et al. 2014). The velocity dispersion of Coma within $R_{200}$ is $\sigma_{c l}=947 \pm 31 \mathrm{~km} \mathrm{~s}^{-1}$, also similar to the previous measurements: $\sigma_{c l}=1082 \pm 74 \mathrm{~km} \mathrm{~s}^{-1}$ (Colless \& Dunn 1996) and $\sigma_{c l}=957_{-28}^{+30} \mathrm{~km} \mathrm{~s}^{-1}$ (Rines et al. 2003).

\section{Luminosity, Stellar Mass, and Velocity Dispersion Function of Cluster Galaxies}

We construct the luminosity, stellar mass, and central velocity dispersion functions for A2029 and Coma. These three functions are powerful probes of the mass distribution of DM subhalos, crucial for modeling galaxy formation and evolution. Conventionally, luminosity and SMFs have been favored because they can be derived from photometric data alone. However, contamination by interlopers can be a serious issue (e.g., Geller et al. 2014). Here, we measure the luminosity (Section 4.1) and SMFs (Section 4.2) based on samples of spectroscopically identified members. We also compute the VDFs for quiescent galaxies (Section 4.3) that may provide a more direct connection to the DM halo masses (see Zahid et al. 2016 and the reference therein).

We note that the observed cluster LFs, SMFs, and VDFs are derived from data taken with a circular aperture on the sky. The cluster members are thus located within a cylinder. As described in Section 3, the caustic technique unavoidably includes a small contamination from interlopers. The analysis 


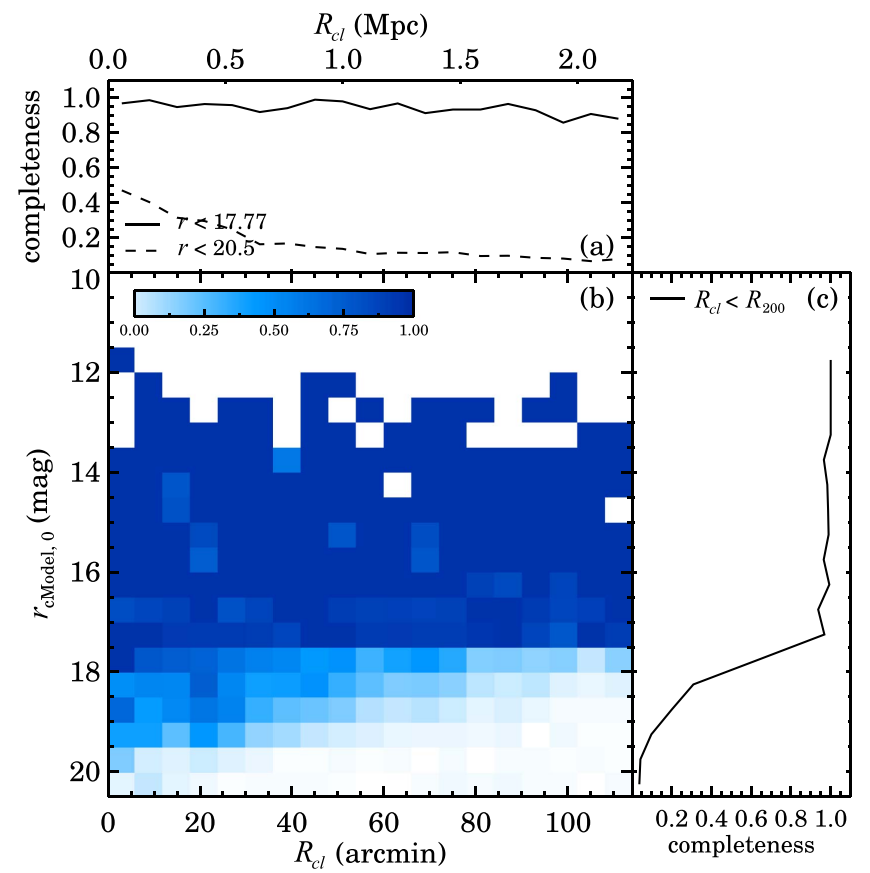

Figure 4. Same as Figure 1, but for Coma. Solid line in the upper panel shows the completeness to $r=17.77$ the SDSS spectroscopic survey limit.

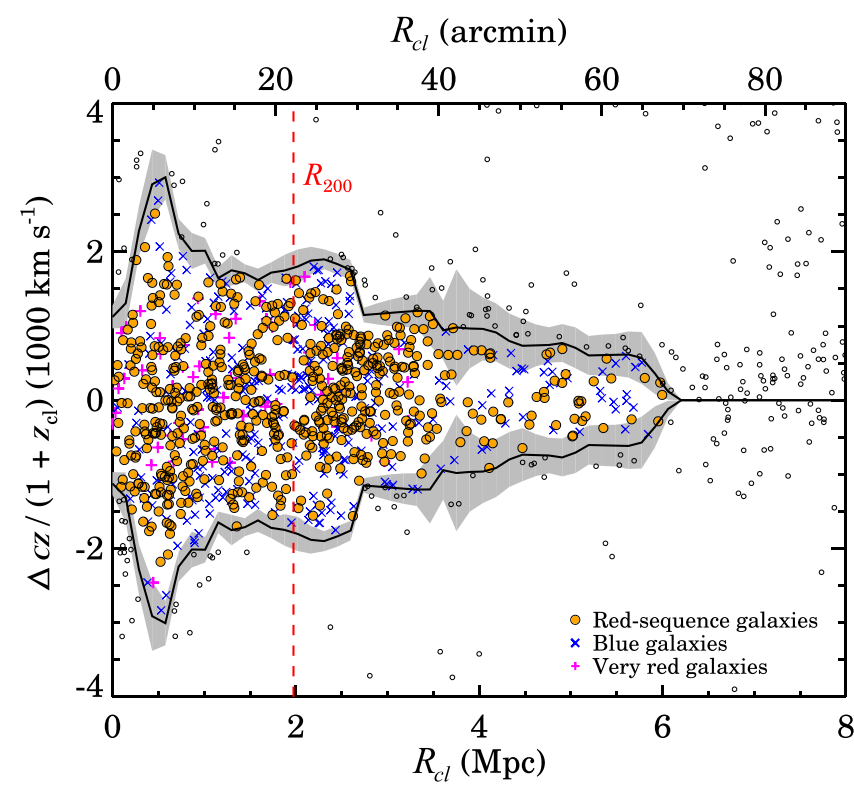

Figure 5. Rest-frame cluster-centric radial velocity vs. projected cluster-centric distance for A2029. Filled circles, crosses, and pluses represent red-sequence, blue, and very red A2029 members, respectively. Open circles indicate A2029 non-members. The solid line shows the caustics for A2029 and the shaded regions shows the uncertainty in the caustic estimate. The vertical dashed line shows $R_{200}$.

also includes objects located outside the projected radius but projected along the line of sight at distances from the cluster center ranging up to the limit of the infall region. For all three functions, we report the observed projected number density per $\mathrm{Mpc}^{2}$ or the count within $R_{200}$. The shapes of these functions are the robust observables.

\subsection{The Spectroscopic Luminosity Function}

At the faint limit, the redshift surveys are not complete (see Figures 2 and 4). Thus, we must correct for spectroscopic incompleteness (Rines \& Geller 2008; Agulli et al. 2014). We follow the method of Rines \& Geller (2008), who correct for missing members as a function of apparent magnitude, projected distance from the cluster center, and galaxy color.

The upper panels of Figure 7 show the member fractions as a function of magnitude (left panel) and projected cluster-centric distance (right panel) for A2029. The member fraction is $f_{\text {member }}=N_{\text {member }} / N_{\text {spec }}$, where $N_{\text {member }}$ is the number of caustic members, and $N_{\text {spec }}$ is the number of spectroscopic targets. We investigate the member fraction trends for redsequence, blue, and very red galaxies, separately (see Figure 1 for classifications). The member fractions decline as a function of magnitude for all three populations (the upper left of Figure 7). However, the member fractions of the three populations behave differently, emphasizing the need for separate corrections. The member fractions also decrease with radius. Again, the member fractions for the three populations differ. The member fraction for the very red population drops more rapidly than the other populations. The lower panels of Figure 7 plot the same quantities for Coma. The member fractions for Coma behave in essentially the same way as those for A2029.

Using the member fractions in Figure 7, we apply corrections to account for missing members. We first count the number of photometric galaxies $\left(N_{\text {phot }}\right)$ in the three populations within 0.5 magnitude bins. Then, we derive the corrected LFs:

$$
\phi\left(m_{r}\right)=N_{\text {phot }}\left(m_{r}\right) \times \frac{N_{\text {member }}\left(m_{r}\right)}{N_{\text {spec }}\left(m_{r}\right)} \times \frac{1}{A},
$$

where $A$ is the area. The total LF is the sum of the LFs for the three populations. We restrict our analysis to $R_{c l}<R_{200}$ where the corrections are relatively small for both clusters.

Figure 8 shows the $r$-band spectroscopic LFs of A2029 and Coma. We compare these results with the spectroscopic LF of the A2199 cluster (Rines \& Geller 2008), another massive cluster in the local universe $\left(z=0.03, \sigma_{c l}=676 \mathrm{~km} \mathrm{~s}^{-1}\right)$. Using the A2199 data from Rines \& Geller (2008), we also measure the LF within $R_{c l}<R_{200}$. We plot the A2199 LF as a sum of the three cluster populations.

The LFs of all three clusters are remarkably similar for $M_{r}<-18$ except for small discrepancies at the very bright end. Coma has a few more bright galaxies at $M_{r}<-22$; these objects are the brightest cluster galaxies (BCGs) within each substructure of Coma (Colless \& Dunn 1996; Okabe et al. 2014). Thus, the increment in the Coma LF at the bright end probably results from the complex nature of the cluster. In contrast, A2029 and A2199 have a single BCG.

Toward the faint end, the three LFs appear to have different shapes. The Coma and A2199 LFs show a slight upturn for $M_{r} \gtrsim-18.0$; the A2029 LF appears to decline. The Coma LF shows an even stronger upturn at the faint end when low surface brightness galaxies, ultra compact dwarfs, and globular clusters are taken into account (Milne et al. 2007).

The A2029 LF appears to decline at the faint end as a result of the surface brightness limit of the spectroscopic sample. A2029 is more distant than both Coma and A2199. Consequently, the observed surface brightness at the same absolute 
Table 2

A2029 Spectroscopic Members

\begin{tabular}{|c|c|c|c|c|c|c|c|}
\hline $\begin{array}{l}\text { R.A. } \\
\text { (J2000) }\end{array}$ & $\begin{array}{c}\text { Decl. } \\
\text { (J2000) }\end{array}$ & $\begin{array}{c}c z \\
\left(\mathrm{~km} \mathrm{~s}^{-1}\right)\end{array}$ & $\begin{array}{c}c z \text { error } \\
\left(\mathrm{km} \mathrm{s}^{-1}\right)\end{array}$ & $\begin{array}{c}\sigma^{\mathrm{a}, \mathrm{b}} \\
\left(\mathrm{km} \mathrm{s}^{-1}\right)\end{array}$ & $\begin{array}{c}\sigma \text { error } \\
\left(\mathrm{km} \mathrm{s}^{-1}\right)\end{array}$ & $D_{n} 4000$ & $\operatorname{Ref}^{c}$ \\
\hline 227.740259 & 5.766147 & 23177 & 29 & 284 & 5 & 2.03 & T13 \\
\hline 227.737793 & 5.762320 & 22763 & 22 & 95 & 17 & 2.03 & $\mathrm{~T} 13$ \\
\hline 227.738249 & 5.754465 & 23162 & 26 & 229 & 10 & 1.86 & $\mathrm{~T} 13$ \\
\hline 227.749463 & 5.769346 & 23468 & 22 & 61 & 14 & 1.91 & $\mathrm{~T} 13$ \\
\hline 227.735039 & 5.751555 & 23797 & 35 & 261 & 42 & 1.91 & MMT \\
\hline 227.735860 & 5.775843 & 24381 & 24 & 151 & 5 & 1.79 & $\mathrm{~T} 13$ \\
\hline 227.728122 & 5.756973 & 24019 & 26 & 160 & 7 & 1.88 & $\mathrm{~T} 13$ \\
\hline
\end{tabular}

Notes. The entire table is available in machine-readable form in the online journal. Here, a portion is shown for guidance regarding its format.

a The central velocity dispersion within a rest-frame $3 \mathrm{kpc}$ aperture.

b -99 indicates lack of a measurement for $\sigma$.

c The redshift source: "SDSS" from SDSS and BOSS, "T13" from Tyler et al. (2013) and "MMT" from the MMT/Hectospec observation for this study.

(This table is available in its entirety in machine-readable form.)

magnitude is significantly fainter in A2029. Figure 9 demonstrates this point. The spectroscopic completeness for A2029 declines rapidly at $M_{r} \sim-18$ because the redshift survey is incomplete at $r_{\text {fib }}>21$.

We fit a Schechter function (Schechter 1976),

$$
\phi(M)=\phi_{0} 10^{0.4(1+\alpha)\left(M_{*}-M\right)} \exp \left[-10^{0.4\left(M_{*}-M\right)}\right]
$$

to the LFs in the range $-22.0<M_{r}<-18.0$. Table 3 summarizes the Schechter function-fitting parameters for Coma, A2199, and A2029. The A2029 spectroscopic LF has $\alpha=-0.81 \pm 0.17$ and $M_{r, *}=-21.00 \pm 0.27$. The LFs of the other two clusters are also well described by Schechter functions: $\alpha=-1.02 \pm 0.11$ and $M_{r, *}=-21.17 \pm 0.30$ for Coma and $\alpha=-0.90 \pm 0.29$ for $M_{r, *}=-21.04 \pm 0.41$ for A2199, respectively. These slopes are consistent with the slope for the spectroscopic LF of A85 ( $z=0.055$, Agulli et al. 2014) with $\alpha=-0.67 \pm 0.25$ at the bright end $-22<M_{r}<-19$.

The shape of a spectroscopic LF may differ from a photometrically determined LF for two reasons: (1) interloper contamination tends to be greater for photometrically selected samples and (2) the fitting range for the LF may not be restricted to the bright end. Laganá et al. (2011) present photometric LFs of these three clusters based on SDSS $i$-band photometry. They fit these LFs with double Schechter functions at the bright and faint ends separately. Their fits for the bright end $\left(M_{i}<-18\right)$ are generally steeper than ours: $\alpha=-1.26 \pm 0.03$ for Coma, $\alpha=-1.18 \pm 0.03$ for A2199, and $\alpha=-1.17 \pm 0.07$ for A2029. The difference may result from interlopers. We note again that $>40 \%$ of the cluster members on the red sequence with $M_{i}<-18$ are non-members. The photometric LFs include some of these interlopers and are thus steeper. The interloper fraction increases for less luminous objects. The photometric LFs of Coma derived by Andreon \& Cuillandre (2002) and Milne et al. (2007) are substantially steeper, but the difference here is dominated by their broader fitting range, $M_{R}<-9.5$.

Steeper spectroscopic LFs for Coma and A2199 in the literature may also result from their broader fitting range: $\alpha \sim-1.2$ for Coma for $M_{R}<-16$ (Mobasher et al. 2003),

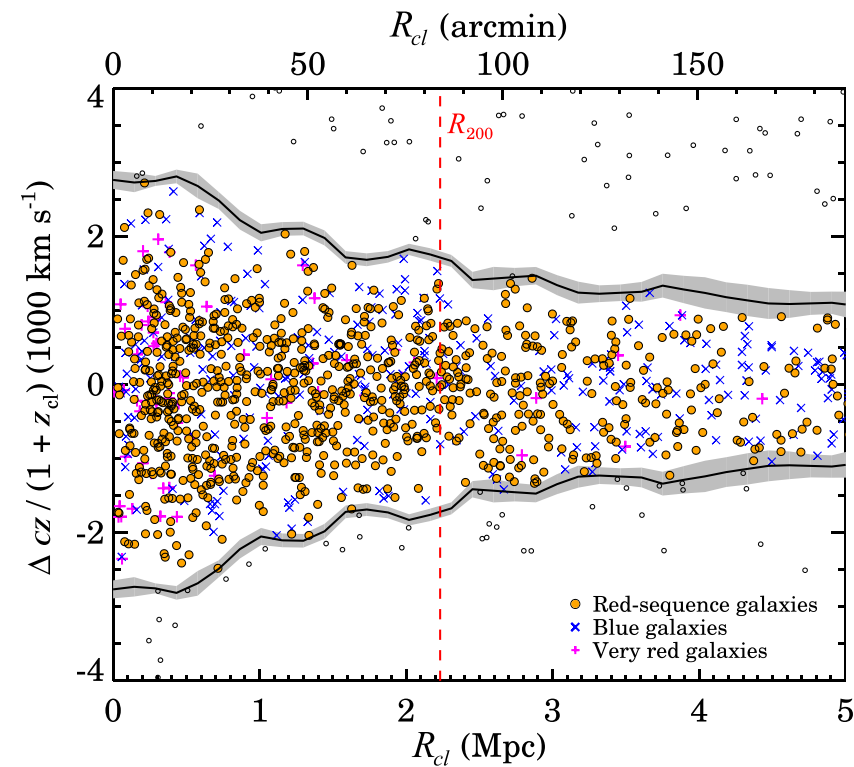

Figure 6. Rest-frame cluster-centric radial velocities vs. projected clustercentric distances for Coma. Symbols and lines are the same as in Figure 5.

$\alpha \sim-1.1$ for A2199 for $M_{r}<-16$ (Andreon 2008; Rines \& Geller 2008). In fact, we derive a similar slope when we fit the A2199 LF to $M_{r}<-16$. The slope dependence on the fitting range is well known (Agulli et al. 2014; Moretti et al. 2015; Lan et al. 2016). Lan et al. (2016) also show that cluster LFs become steeper faintward of $M_{r}>-18$. This behavior is evident in Figure 8.

\subsection{Stellar Mass Function}

The SMF appears to be a more fundamental tracer of DM halo masses than the LF (More et al. 2011; Li et al. 2013). However, SMFs are less frequently measured because derivation of the stellar mass requires multi-band photometry. Furthermore, stellar mass is not directly observable. The 

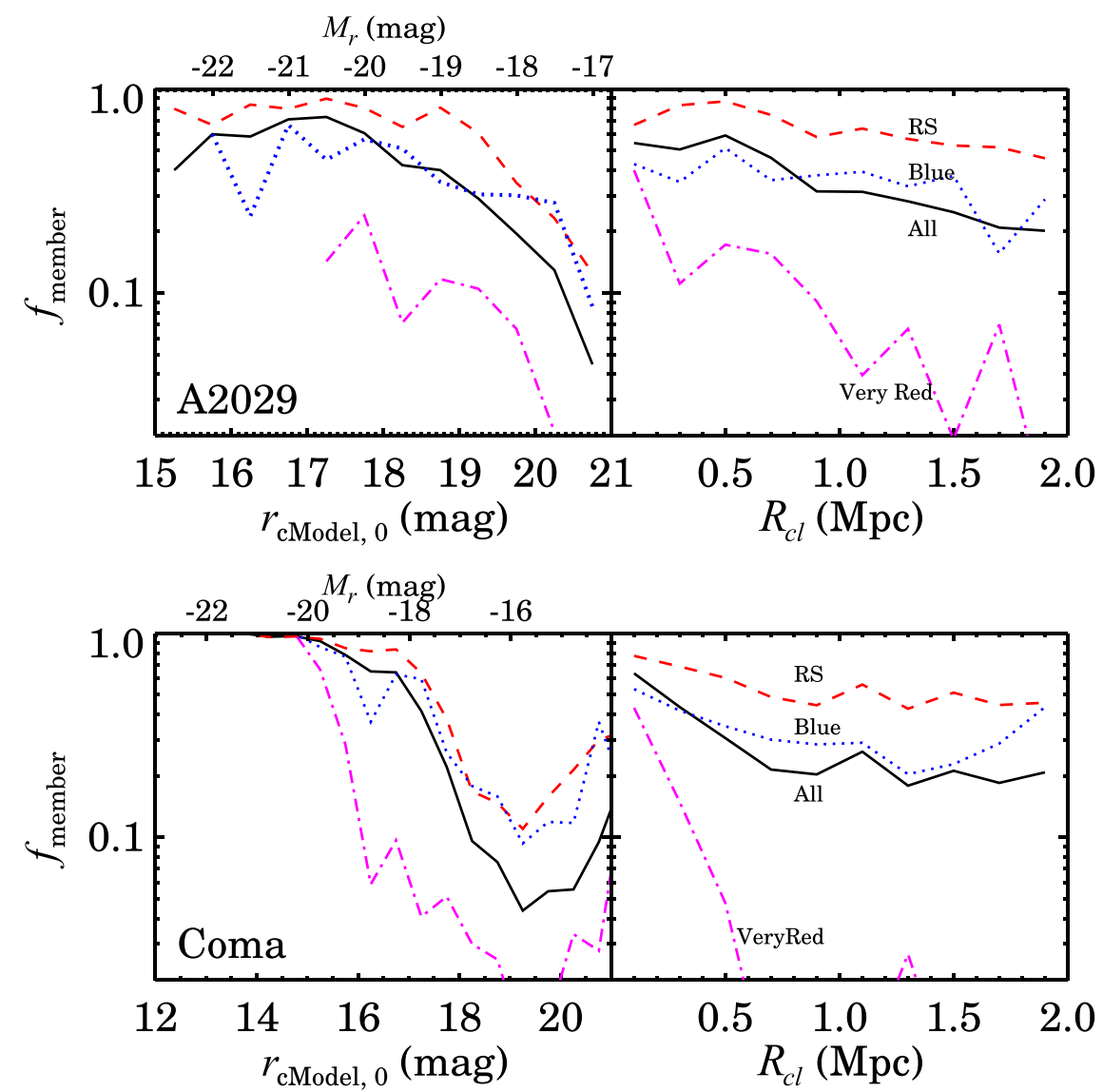

Figure 7. (Upper panels) Spectroscopic member fractions for A2029 as a function of magnitude (left) and cluster-centric distance (right). Lines represent the fractions for red-sequence galaxies (dashed), blue (dotted), and very red (dotted-dashed) galaxies. Solid lines show the sum of the three populations. (Lower panels) Same as the upper panels, but for Coma. Note that the behavior of three populations is similar for the two clusters.

derived stellar mass is sensitive to the stellar IMF and star formation history (Conroy et al. 2009). Thus, comparison of SMFs for different clusters must be based on consistent stellar mass computations.

To compute complete cluster SMFs, we correct for stellar masses of two types of missing members. First, stellar mass estimation miscarries for 5\% of the members in A2029 and 2\% of the members in Coma, respectively. Second, there are missing members resulting from spectroscopic incompleteness (see Figure 7).

We empirically estimate the stellar masses $\left(M_{*}\right)$ for both types of missing members based on the conditional probability distributions $P\left(M_{*} \mid M_{r}\right)$ of $M_{*}$ given the absolute magnitude for the three-different galaxy populations. The conditional probability distribution $P\left(M_{*} \mid M_{r}\right)$ depends on galaxy type. Thus, we derive these distributions empirically for red-sequence, blue, and very red galaxies based on $k$-corrected colors for galaxies in the wide-field SDSS for the redshift range $0.01<z<0.09$ where the upper redshift limit is the A2029 redshift. We identify the red-sequence in the $(g-r)-r$ color-magnitude diagram.

Figure 10 shows the conditional probability distributions $P_{r S}\left(M_{*} \mid M_{r}\right)$ for SDSS red-sequence galaxies in different fixed magnitude ranges. The $M_{*}$ distribution for each magnitude bin is well represented by a Gaussian distribution. The dashed lines in Figure 10 show the best-fit Gaussian model for the set of
$P_{r S}\left(M_{*} \mid M_{r}\right)$ distributions. The distributions, $P_{\text {blue }}\left(M_{*} \mid M_{r}\right)$ and $P_{v r}\left(M_{*} \mid M_{r}\right)$ for SDSS blue and very red galaxies, respectively, can also be described by Gaussian models. However, the means and widths of the Gaussian distributions differ for different populations. For example, the dotted blue lines in Figure 10 show the best-fit Gaussian distributions for the set of $P_{\text {blue }}\left(M_{*} \mid M_{r}\right)$. This comparison clearly shows the necessity of an empirical correction that depends on both color and magnitude.

Using the SDSS field samples for the three populations covering the relevant magnitude range, we compute the entire set of distributions, $P_{\text {pop }}\left(M_{*} \mid M_{r}\right)$. For members where the $M_{*}$ computation failed, we know the absolute magnitude. For members missing as a result of spectroscopic incompleteness, we randomly select a galaxy in the appropriate apparent magnitude bin and then, because we take the object as a cluster member, we automatically have the absolute magnitude. In both cases, we derive an $M_{*}$ for the galaxy by drawing randomly from the appropriate $P\left(M_{*} \mid M_{r}\right)$. We repeat the process for the entire sample of missing members 1000 times. Each of these 1000 samples provides an estimate of the SMF. We then take the mean of these 1000 SMFs as the "corrected" SMF of the cluster.

Figure 11 displays the SMFs of Coma and A2029 for $R_{c l}<R_{200}$. The dotted and solid lines show the raw and corrected SMFs, respectively. The corrections are significant 


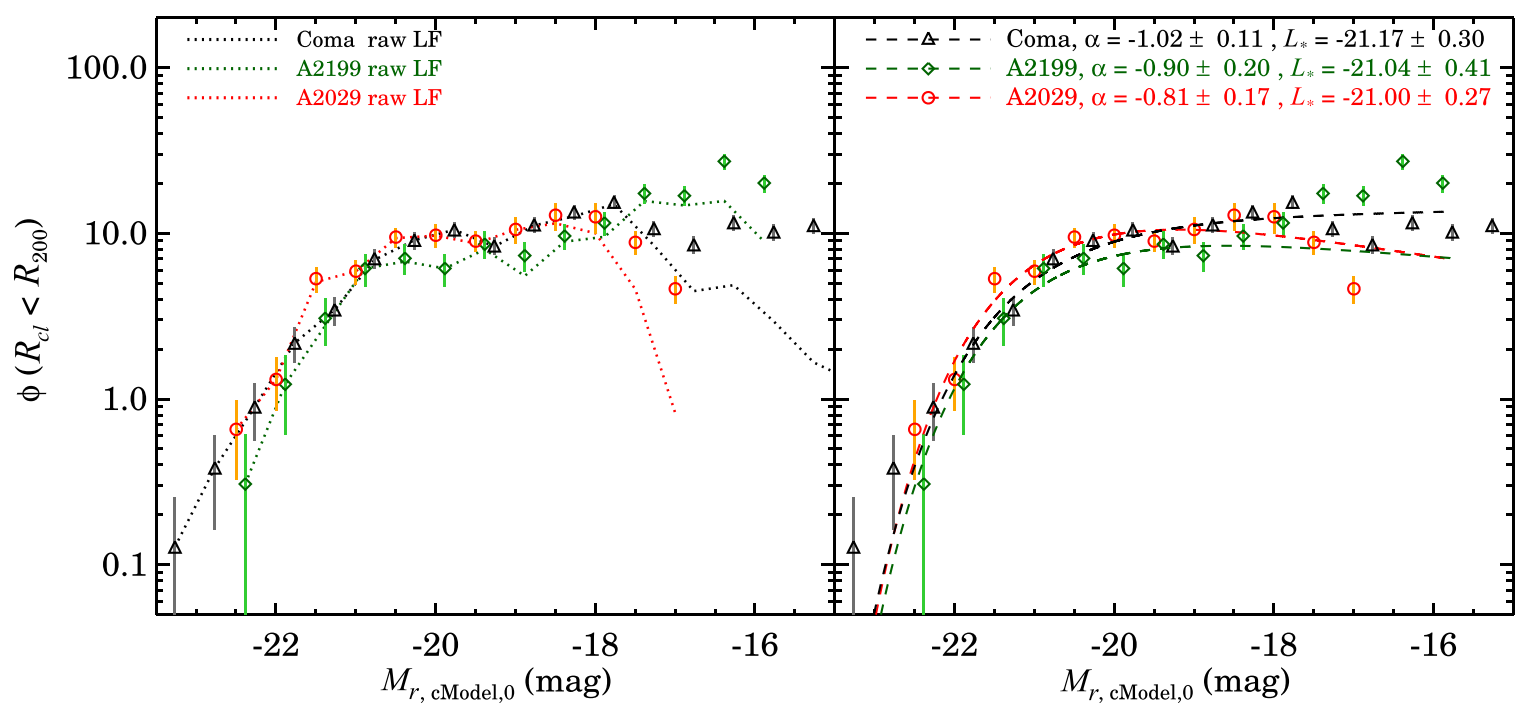

Figure 8. Spectroscopic luminosity function of A2029 (circles), Coma (triangles), respectively, corrected for incompleteness. For reference, the diamonds show the LF for A2199 (Rines \& Geller 2008). The left panel shows the raw luminosity functions (dotted lines). The right panel displays the Schechter function fits for the magnitude range $-22<M_{r, \text { cModel }, 0}<-18$.

only for $\log \left(M / M_{\odot}\right)<9.5$ corresponding to $M_{r}<-18.5$, where the spectroscopic completeness declines.

The two SMFs are similar for $9.5 \leqslant \log \left(M_{*} / M_{\odot}\right) \leqslant 11.5$ where the corrections for incompleteness are negligible. As for the LF, the A2029 SMF appears to decline rapidly at low $M_{*}$, but the Coma SMF remains flat. This difference is an artifact resulting from the relatively larger spectroscopic incompleteness of the A2029 sample at faint magnitudes.

We fit the SMFs with the Schechter form in the range $9.5 \leqslant \log \left(M_{*} / M_{\odot}\right) \leqslant 11.5$. The results for Coma and A2029 MFs are $\alpha=-1.04 \pm 0.04, M_{*}=10.65 \pm 0.06$ for Coma and $\alpha=-0.97 \pm 0.13, M_{*}=10.69 \pm 0.14$ for A2029. The two SMFs are consistent. The MFs of these clusters are somewhat steeper than their LFs, but the $M_{*}$ values are consistent with a direct conversion of the luminosity into $M_{*}$ using the relation from the SDSS field galaxies on the redsequence: $M_{* \text {,converted }} \sim 10.52$ for Coma and $M_{* \text {,converted }} \sim$ 10.48 for A2029.

Figure 10 demonstrates that overall construction of the SMF by converting mean luminosities to mean $M_{*}$ is inadequate. In a fixed magnitude range, the $M_{*}$ distribution depends on galaxy type or color. Furthermore, the $M_{*}$ range varies with the range of absolute magnitude. At the bright end, the LF is dominated by the red-sequence population which has a narrow $M_{*}$ distribution with a width increasing slowly with magnitude. In contrast, blue galaxies that contribute increasingly at fainter magnitudes have broader $M_{*}$ distributions. These broader distributions steepen the low mass end of the SMF relative to the slope of the LF.

There are only a few published cluster SMFs extending to $\log \left(M_{*} / M_{\odot}\right) \sim 10.0$. Vulcani et al. (2011) obtain a similar slope, $\alpha=-0.987 \pm 0.009$, for cumulative SMFs for the low- $z$ WINGS cluster sample (Fasano et al. 2006). They obtain $\alpha=-0.915 \pm 0.026$ for the SMFs of the EDisCS sample (White et al. 2005). Vulcani et al. (2013) also report similar slopes from cluster samples at redshift $0.3<z<0.8$.

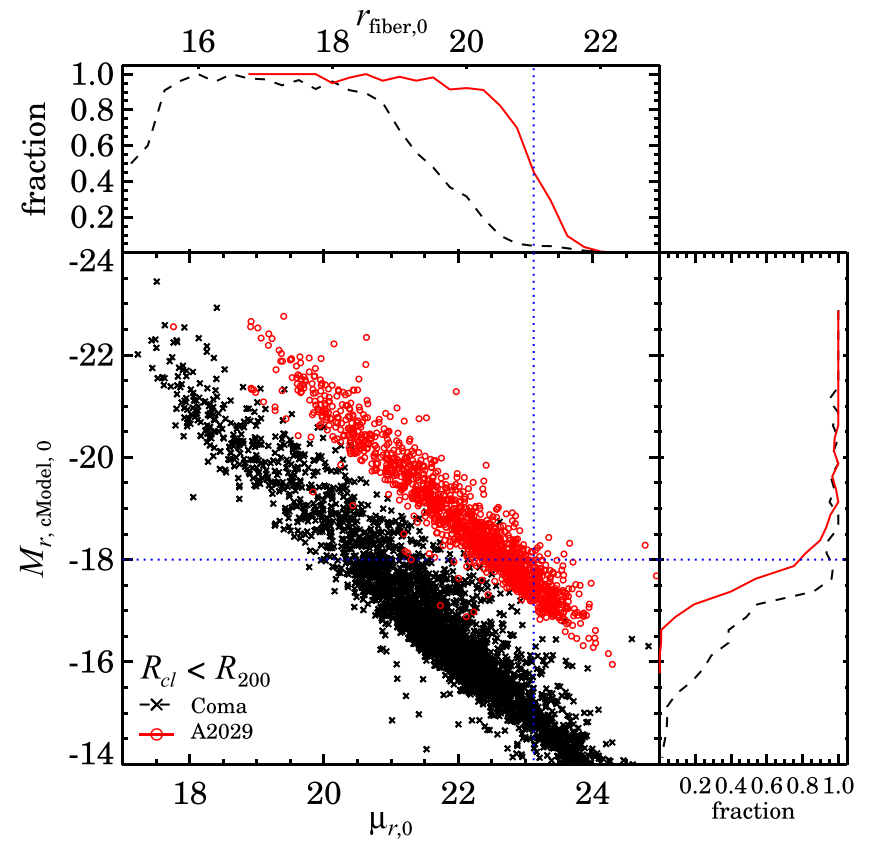

Figure 9. Absolute magnitude vs. surface brightness for the spectroscopic samples in the Coma (crosses) and A2029 fields (circles). The upper panel shows the spectroscopic completeness as a function of surface brightness. Dashed and solid lines show the completeness for Coma and A2029, respectively. The right-hand panel displays the completeness but as a function of absolute magnitude.

\subsection{Velocity Dispersion Function}

The central velocity dispersion of a galaxy reflects the stellar kinematics. The central velocity dispersion is a stellar luminosity-weighted sum over objects within the fiber aperture. We correct this observed dispersion to a $\sigma$ measured within a $3 \mathrm{kpc}$ radius (see Section 2.1.4). This dispersion may be proportional to the dispersion of the DM halo and thus it may be a fundamental observable for studying the DM halo distribution (see Zahid et al. 2016). The velocity dispersion is 
Table 3

The Spectroscopic Luminosity Function Parameters

\begin{tabular}{|c|c|c|c|c|}
\hline Cluster & Fitting range & $\alpha$ & $M_{*}$ & Ref. \\
\hline A2029 & $-22.0 \leqslant M_{r} \leqslant-18.0$ & $-0.81 \pm 0.17$ & $-21.00 \pm 0.27$ & This study \\
\hline Coma & $-22.0 \leqslant M_{r} \leqslant-18.0$ & $-1.02 \pm 0.11$ & $-21.17 \pm 0.30$ & This study \\
\hline A2199 & $-22.0 \leqslant M_{r} \leqslant-18.0$ & $-0.90 \pm 0.29$ & $-21.04 \pm 0.41$ & This study \\
\hline A2199 & $-22.5 \leqslant M_{r} \leqslant-16.0$ & $-1.13 \pm 0.07$ & $-21.11 \pm 0.25$ & Rines \& Geller (2008) \\
\hline Virgo & $-22.0 \leqslant M_{r} \leqslant-16.5$ & $-1.28 \pm 0.06$ & -21.32 & Rines \& Geller (2008) \\
\hline A85 & $-22.5 \leqslant M_{r} \leqslant-19.0$ & $-0.79 \pm 0.09$ & $-20.85 \pm 0.14$ & Agulli et al. (2014) \\
\hline
\end{tabular}

a reasonable halo mass proxy for early-type galaxies dominated by random motions (Wake et al. 2012; van Uitert et al. 2013; Bogdán \& Goulding 2015; Zahid et al. 2016). For late-type galaxies, the circular velocity is important for characterizing the disk (Sheth et al. 2003).

We examine the VDF only for quiescent cluster members with $D_{n} 4000>1.5$. This criterion conservatively identifies early-type galaxies mainly consisting of an older stellar population (Kauffmann et al. 2003; Woods et al. 2010; Zahid et al. 2016). Previous studies of VDFs for field galaxies (e.g., Sheth et al. 2003; Choi et al. 2007; Montero-Dorta et al. 2016) use a variety of definitions for early-type galaxies. Here we use a homogeneous spectroscopic definition. Moresco et al. (2013) examine the dependence of the properties of quiescent galaxies in the zCOSMOS-20k spectroscopic sample on the quiescent galaxy selection algorithm. They show that each classification method yields somewhat different subsamples of galaxies, but the overall properties of the quiescent galaxy samples are insensitive to the classification scheme.

Obtaining a $\sigma$ depends strongly on the signal-to-noise ratio of each spectrum. Thus, we lack a $\sigma$ measurement for some cluster members, even though we have a reliable redshift. Furthermore, there are a few $\sigma$ measurements with very large uncertainty. Hereafter, we use only $\sigma$ s with an error $<100 \mathrm{~km} \mathrm{~s}^{-1}$. Figure 12 shows the completeness of $\sigma$ measurements for A2029 and Coma members. The $\sigma$ completeness for quiescent members is much higher than for the entire sample. For quiescent galaxies with $D_{n} 4000>1.5$, the $\sigma$ measurements are $>80 \%$ complete to $M_{r}<-19$ for A2029 and $>80 \%$ complete to $M_{r}<-19.5$ for Coma.

Figure 13 displays $\sigma$ as a function of $M_{*}$ for A2029 and Coma members. For comparison, we plot the relation for field galaxies derived by Zahid et al. (2016) using a local SDSS sample $(0.0<z<0.2)$ and an intermediate redshift $(0<z<0.7)$ sample from the Smithsonian Hectospec Lensing Survey (SHELS; Geller et al. 2005, 2014, 2016) F2 field. Zahid et al. (2016) show that, for massive galaxies, the slope and zero-point of the $M_{*}-\sigma$ relation does not significantly evolve at $z<0.3$. Although the redshift ranges of the two field samples differ, the slopes at high $M_{*}\left(\log \left(M_{*} / M_{\odot}\right)>10.2\right)$ are similar.

The cluster galaxies follow the trend of field galaxies except at the very massive end, where some outliers in the cluster samples lie above the trend for field galaxies, i.e., these cluster objects have larger $\sigma$ at a given $M_{*}$. These outliers with $D_{n} 4000 \gtrsim 2.0$ consist of an older stellar population and may be metal rich. Field samples also contain galaxies with $D_{n} 4000>2.0$ (Figure 1 in Zahid et al. 2016), but they are rare. These large $D_{n} 4000$ field galaxies also tend to have larger $\sigma$ at a fixed stellar mass (Zahid \& Geller 2017). The offset between cluster and field galaxies at the massive end reflects differences in the $D_{n} 4000$ distributions of cluster and field samples.
As for the SMFs, we correct the VDF for two types of missing members. First, we lack $\sigma$ measurements for $\sim 15 \%$ of A2029 members and $\sim 18 \%$ of Coma members, respectively. These members have reliable redshifts, but the spectrum is not available. There are also missing $\sigma$ s for probable members resulting from spectroscopic incompleteness.

We derive $\sigma$ for missing members based on conditional probability distributions of $\sigma$ given the absolute magnitude and $D_{n} 4000, P\left(\sigma\left|D_{n} 4000\right| M_{r}\right)$. As shown in Figure 13 , the conditional probability distribution of $\sigma$ depends strongly on $D_{n} 4000$. We derive these distributions empirically for SDSS galaxies in different $D_{n} 4000$ ranges. We set five bins with $1.5<D_{n} 4000 \leqslant 2.0$ with bin size of 0.1 and we reserve one bin for $2.0<D_{n} 4000<3.0$. The bin sizes are chosen to have similar numbers of galaxies in each bin. We also determine the probability distribution for the entire SDSS sample with $D_{n} 4000>1.5$. We use this probability distribution for cluster members lacking a $D_{n} 4000$ and a $\sigma$ measurement.

Figure 14 shows the conditional probability distributions $P\left(\sigma\left|1.5<D_{n} 4000 \leqslant 1.6\right| M_{r}\right)$ for SDSS galaxies in different fixed magnitude ranges and fixed $D_{n} 4000$ ranges. The dashed lines in Figure 14 represent the best-fit Gaussian model for the set of $P\left(\sigma\left|1.5<D_{n} 4000 \leqslant 1.6\right| M_{r}\right)$ distributions. The distributions are skewed to low $\sigma$ in some magnitude bins. However, we ignore the contribution of low $\sigma$ objects because the $\sigma$ measurement for SDSS galaxies are reliable only to $\sigma \sim 60 \mathrm{~km} \mathrm{~s}^{-1}$ (Thomas et al. 2013). The distributions for the other $D_{n} 4000$ bins can also be fit with Gaussian models, but with different means and widths. Note that the widths of the conditional probability distributions, $\sim 40 \mathrm{~km} \mathrm{~s}^{-1}$ at mean $\sigma$ of $100 \mathrm{~km} \mathrm{~s}^{-1}$, are larger than typical uncertainty in the $\sigma$ measurements for both the SDSS and Hectospec samples. We use the set of best-fit Gaussian distributions to construct a set of $P\left(\sigma\left|D_{n} 4000\right| M_{r}\right)$ based on the SDSS field samples.

We compute $\sigma$ for missing members using the set of $P\left(\sigma\left|D_{n} 4000\right| M_{r}\right)$. We know the absolute magnitudes for each member or probable member without a $\sigma$ measurement. For members missing because of spectroscopic incompleteness, we randomly select a galaxy in the appropriate magnitude bin. We calculate $\sigma$ for members and probable members by drawing randomly from the appropriate $P\left(\sigma\left|D_{n} 4000\right| M_{r}\right)$. We repeat the process for all missing members 1000 times and measure the VDF using each of these 1000 samples. Finally, we take the mean of the 1000 VDFs as the "corrected" VDF of the cluster.

Figure 15 shows the corrected VDFs for A2029 and Coma. The left panel of Figure 15 compares the VDFs with the raw VDFs. The corrections for missing members are negligible for $\log \sigma>1.9$ and become significant at lower $\sigma$.

Because the $\sigma$ distribution at each $D_{n} 4000$ and $M_{r}$ is broad, it is critical to reconstruct the VDF by drawing from the conditional probability distributions (Sheth et al. 2003). To 


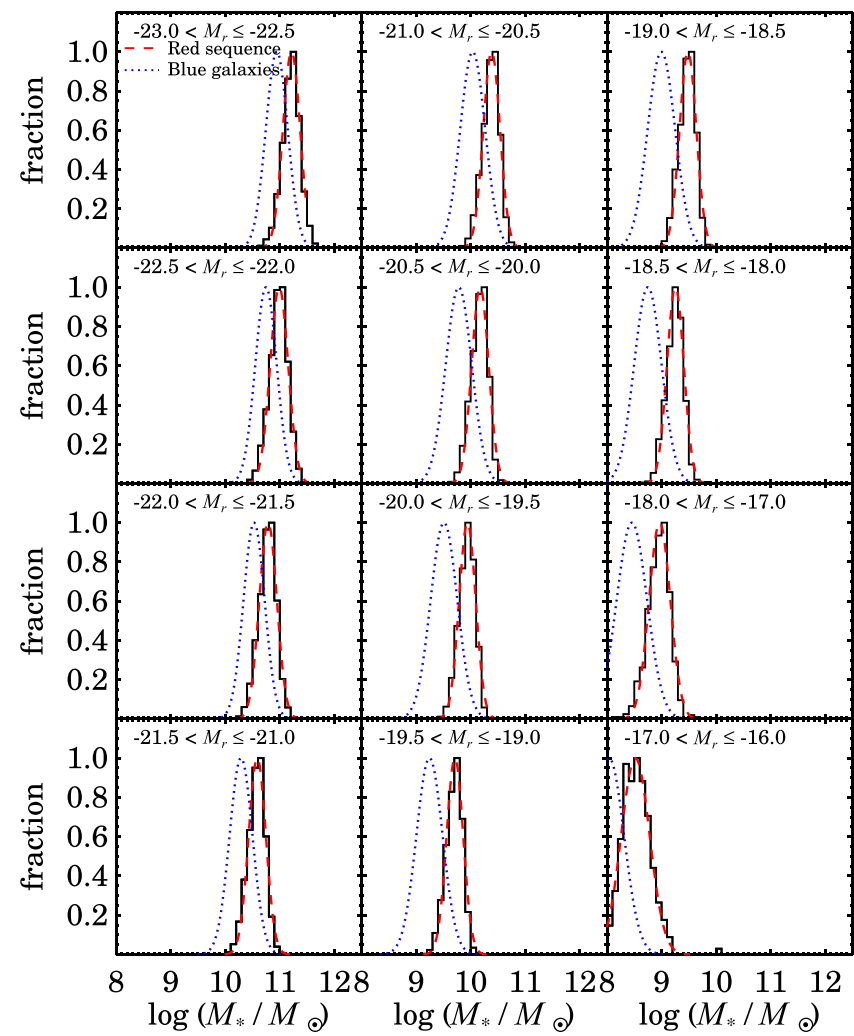

Figure 10. Conditional probability $P_{R S}\left(M_{*} \mid M_{r}\right)$ histograms for red-sequence galaxies derived from the SDSS field sample. The dashed and dotted lines show Gaussian fits for the probability distributions for red sequence galaxies and for blue galaxies. These distributions demonstrate the necessity of correcting separately for the different populations.

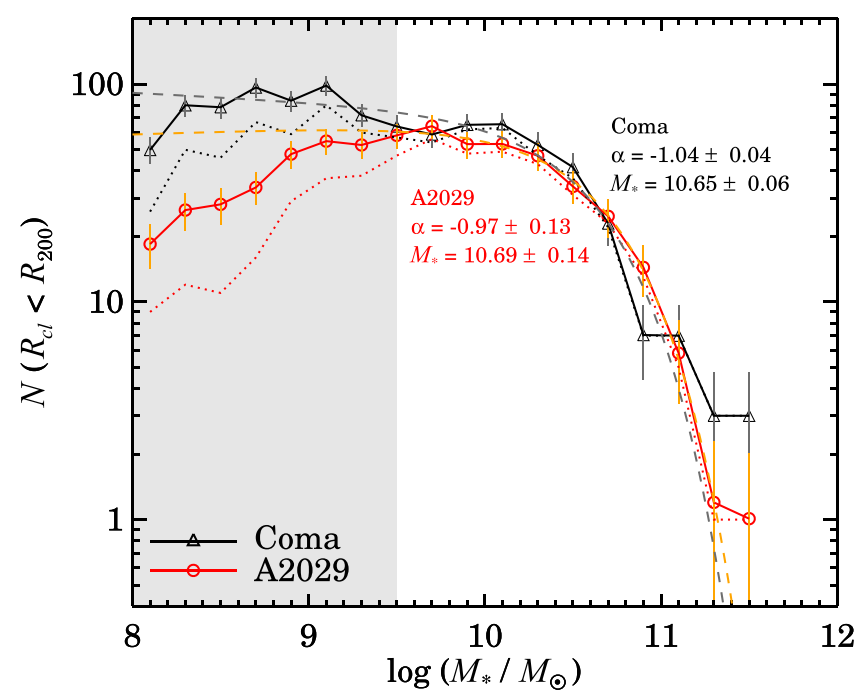

Figure 11. Stellar mass function for Coma (triangles) and A2029 (circles). Error bars are Poisson. The dotted lines show the raw mass function and the dashed lines represent the Schechter function fits for each cluster.

demonstrate this issue, we compare the corrected VDF with a VDF derived by taking the mean $\sigma$ for each absolute magnitude (right panel of Figure 15). Here we estimate the mean $\sigma$ as a function of absolute magnitude using the SDSS field sample with $D_{n} 4000>1.5$. We calculate the mock $\sigma$ for all A2029 and Coma members according to their absolute magnitude. The converted VDFs differ from the observed VDFs in the sense emphasized by Sheth et al. (2003). As Sheth et al. (2003) demonstrate, the direct conversion of $\sigma$ from the absolute magnitudes introduces a strong biases in the $\sigma$ distribution.

As a test of our correction method, we also generate mock cluster VDFs using the set of $P\left(\sigma\left|D_{n} 4000\right| M_{r}\right)$. For these mock VDFs, we draw $\sigma$ for every cluster member. These mock VDFs are very similar to the corrected VDFs. This test substantiates the correction we apply based on the $P\left(\sigma\left|D_{n} 4000\right| M_{r}\right)$ to compensate for missing $\sigma \mathrm{s}$.

The VDFs of A2029 and Coma are essentially identical for $\log \sigma>2.0$, where the corrections for incompleteness are negligible. The remarkably identical shapes of two cluster VDFs are consistent with the similar shapes of the LFs and SMFs. The larger difference between the two VDFs toward low $\sigma$ probably results from the relatively larger spectroscopic incompleteness of A2029.

The similarity of the Coma and A2029 VDFs for $\log \sigma>2.0$ suggests that the underlying DM subhalo mass distributions of the two target clusters are similar. Further studies based on larger cluster samples with different redshift, mass, and dynamical stage may thus provide interesting new probes of the subhalo mass distribution and its evolution.

\section{Discussion}

Taking advantage of an intensive spectroscopic survey based on SDSS and Hectospec observations, we measure the LFs, SMFs, and VDFs for Coma and A2029. There are several systematic issues in determining the shape of these functions. One critical example is the conditional probability distribution functions we use for correcting SMFs and VDFs. Because $M_{*}$ and $\sigma$ depend on galaxy properties including colors and $D_{n} 4000$ (Figures 10 and 14), a direct translation from absolute magnitude to a mean $M_{*}$ or a mean $\sigma$ introduces significant systematic biases. Use of the conditional probability distribution has a more critical impact on the VDF than on the SMF because the typical spread in $P\left(\sigma\left|D_{n} 4000\right| M_{r}\right)$ is much larger than for $P\left(M_{*} \mid M_{r}\right)$. The differences in the conditional probability distribution result in a substantially different shape for the VDFs relative to the LFs. In contrast, the shapes of the SMFs are similar to LFs. We examine other systematic effects on the VDF in Section 5.1.

Comparing observed SMFs and VDFs with simulations tests our understanding of galaxy properties in dense environments. In Section 5.2, we compare our observed SMFs to the SMFs derived from numerical simulations (Behroozi et al. 2013; Lim et al. 2017). These simulations provide appropriate model SMFs measured for mock clusters with similar mass to Coma and A2029. We compare the observed VDFs for quiescent galaxies only with other observations (Section 5.3) because current simulations do not compute the VDF in a way that mimics the observations directly. In particular, to compare directly to observations, model predictions of VDFs need to be made for the luminosity-weighted velocity dispersion within a projected cylinder of fixed aperture. Unfortunately, no such model predictions are currently available.

\subsection{Systematic Effects}

At face value, the measurement uncertainties of $\sigma$ for Hectospec spectra are larger than for SDSS spectra $\left(17 \mathrm{~km} \mathrm{~s}^{-1}\right.$ and $7 \mathrm{~km} \mathrm{~s}^{-1}$, respectively). However, these measurement uncertainties are strongly correlated with the $\mathrm{S} / \mathrm{N}$ ratio, and 


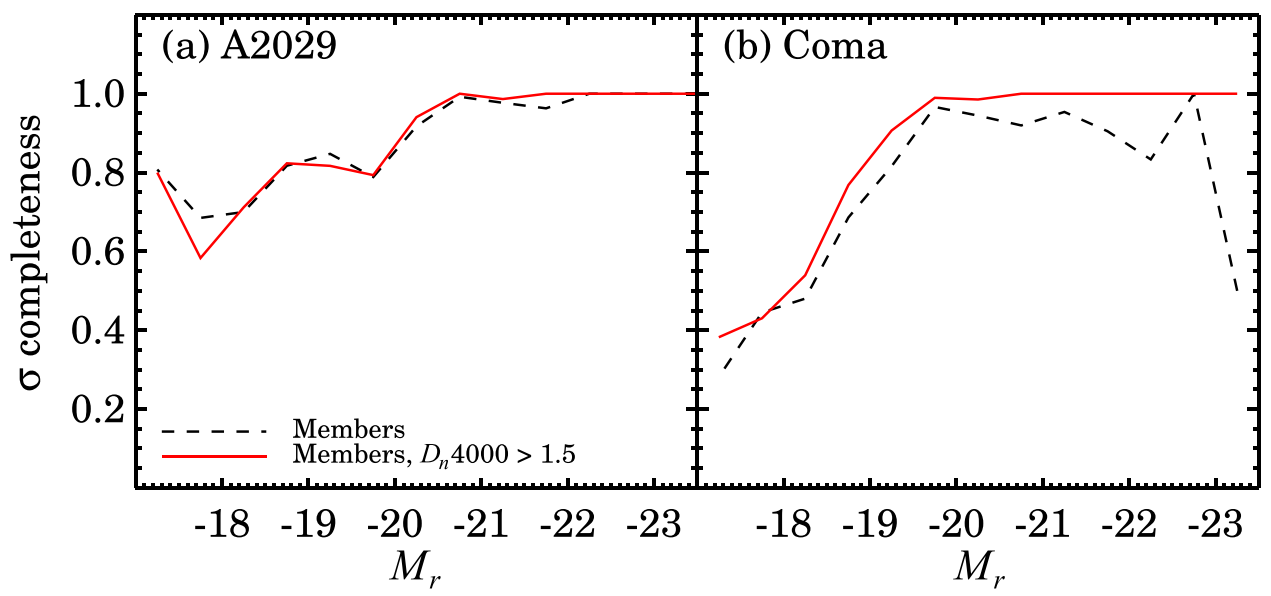

Figure 12. The fractional completeness of $\sigma$ measurements for (a) A2029 members and (b) Coma members. The solid lines and the dashed lines represent all members and quiescent members with $D_{n} 4000>1.5$, respectively.

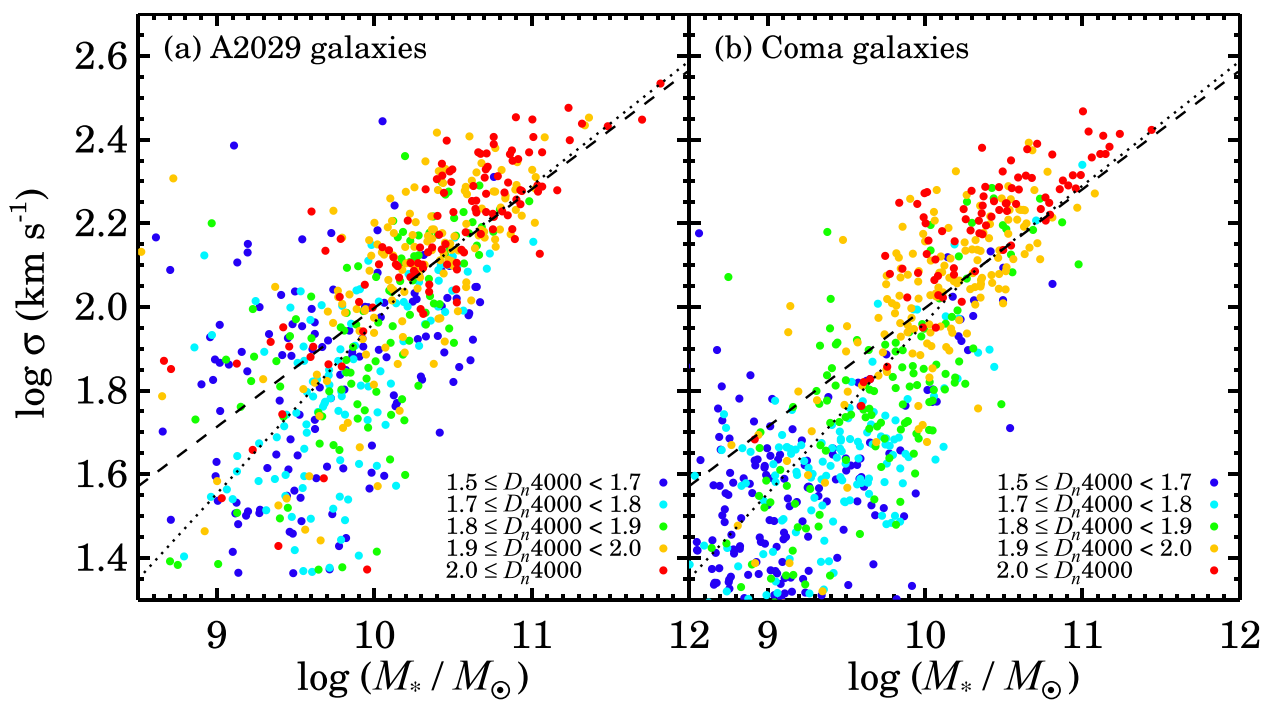

Figure 13. Central velocity dispersion ( $\sigma$ ) vs. stellar mass for (a) A2029 members and (b) Coma members. The color displays $D_{n} 4000$. Dashed and dotted lines show the relation derived for SHELS and SDSS (Zahid et al. 2016).

thus they are also correlated with apparent magnitude. When we compare $\sigma$ measurement errors for A2029 members with $r<17.77$, the typical uncertainties for SDSS and Hectospec are identical $\left(\sim 7 \mathrm{~km} \mathrm{~s}^{-1}\right)$. Larger $\sigma$ measurement errors for Hectospec apply to fainter targets. These fainter Hectospec targets are mostly galaxies with $\sigma \lesssim 100 \mathrm{~km} \mathrm{~s}^{-1}$ where the A2029 VDF becomes incomplete. Thus, measuring the cluster VDF based on the data from two different instruments does not affect the shape of VDF at $\sigma>100 \mathrm{~km} \mathrm{~s}^{-1}$.

Despite the systematic differences in the observations, the A2029 VDF is essentially identical to the Coma VDF. The aperture correction we apply provides a consistent $\sigma$ for A2029 and Coma members. Because Coma members are generally brighter than A2029 members, the typical error in $\sigma$ for Coma members $\left(\sim 4 \mathrm{~km} \mathrm{~s}^{-1}\right)$ is slightly smaller than for A2029 members. These differences in $\sigma$ measurement errors for Coma and A2029 members have a negligible effect on the shape of VDFs.

Figure 14 demonstrates that the larger $\sigma$ measurement errors for the faint targets or A2029 members have little impact on determining the shape of VDF. The $\sigma$ distribution for quiescent galaxies with a fixed $D_{n} 4000$ and $M_{r}$ range has an intrinsic dispersion that is significantly larger than the typical $\sigma$ measurement errors for data from both SDSS and Hectospec. For example, at $M_{r} \sim-20$, the typical measurement uncertainty is 0.05 in $\sigma \sim 100 \mathrm{~km} \mathrm{~s}^{-1}$, while the intrinsic width of the conditional probability distribution is $\sim 0.15$ in $\sigma \sim 100 \mathrm{~km} \mathrm{~s}^{-1}$. In other words, the intrinsic spread of $\sigma$ for quiescent galaxies is a more important determinant of the shape of VDFs than the measurement error.

To correct for missing $\sigma$ s for cluster members, we derive the $P\left(\sigma\left|D_{n} 4000\right| M_{r}\right)$ using the SDSS field sample. The $D_{n} 4000$ distribution for the field actually differs somewhat from the one for cluster members. Figure 16 shows $D_{n} 4000$ versus $M_{r}$ for A2029 members compared with the SDSS field sample (contour). The SDSS field sample lacks galaxies with $M_{r}<-19$ and $D_{n} 4000>1.8$; most cluster members without $\sigma$ measurements appear in this domain. However, the lack of appropriate $P\left(\sigma\left|D_{n} 4000\right| M_{r}\right)$ for these missing members has little effect on the shape of VDFs. Missing cluster members with $M_{r}<-19$ and $D_{n} 4000>1.8$ tend to have $\sigma<100 \mathrm{~km} \mathrm{~s}^{-1}$. Thus the correction is insignificant for $\sigma>100 \mathrm{~km} \mathrm{~s}^{-1}$ (left-hand panel of Figure 15), where we examine the shape of the cluster VDFs. 


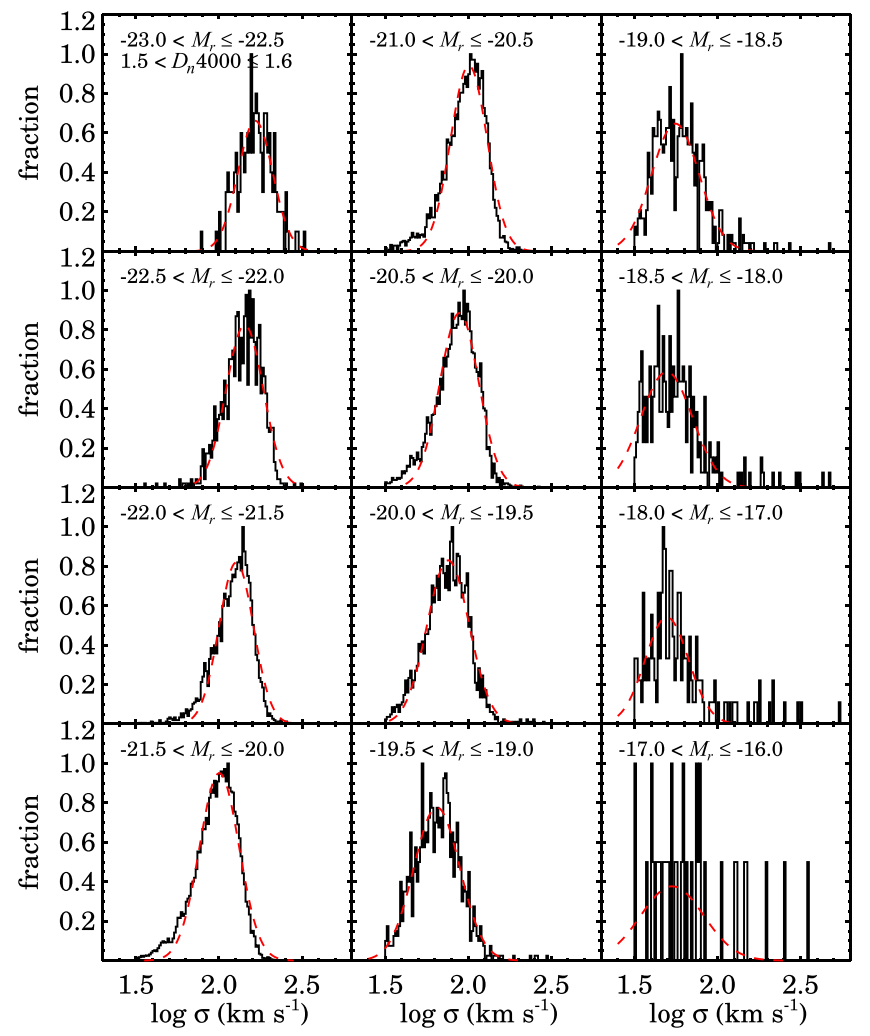

Figure 14. Central velocity dispersion $(\sigma)$ distribution for SDSS field galaxies with $1.5<D_{n} 4000 \leqslant 1.6$ for various absolute magnitude ranges. The dashed lines show Gaussian fits to the $\sigma$ distributions.

\subsection{Comparison of the SMFs with Simulations}

An accurate determination of the LF, SMF, and VDF provides important constraints on galaxy formation models. Traditionally, the LF has been compared to the DM subhalo mass distribution. Ferrarese et al. (2016) show that the Virgo LF (and also SMF) is significantly shallower than the expected distribution from $\Lambda \mathrm{CDM}$ ( $\alpha \sim-1.9$, e.g., Springel et al. 2008). Other spectroscopic LFs including A85 (Agulli et al. 2016) and A2199 (Rines \& Geller 2008) are also less steep than the subhalo mass distributions derived from simulations. However, the complexity of the transformation from subhalo mass to luminosity is nontrivial and thus these comparisons are hard to interpret.

SMFs provide a somewhat more direct basis for comparison with the models. Unlike the LF, the SMF attempts to correct for variations in the stellar populations of galaxies. Lim et al. (2017) compare empirical models of SMFs with observed SMFs in groups/clusters of different halo masses (Lan et al. 2016). For massive clusters with halo mass $\log \left(M_{\text {halo }} / M_{\odot}\right)>13.7$, the empirical models are consistent with the observed SMFs. They also compare the SMFs obtained from hydrodynamic simulations, i.e., Illustris (Vogelsberger et al. 2014) and EAGLE (Schaye et al. 2015); these simulations do not appear to agree as well with the observed SMFs (see Figure 5 of Lim et al. 2017). This result is not surprising because the empirical models of SMFs are constructed to explain the observed SMFs. For the two "ab initio" models, the EAGLE simulation tunes the parameters to match local observations whereas the Illustris simulations include no tuning.
In Figure 17, we compare the Coma and A2029 SMFs with the empirical model SMFs from Lim et al. (2017). For simplicity, we employ two empirical model SMFs from Behroozi et al. (2013) and Lim et al. (2017) (both kindly provided by S.H. Lim) for a halo mass in the range $14.7<\log \left(M_{\text {halo }} / M_{\odot}\right)<15.1$ corresponding to the Coma and A2029 dynamical masses (i.e., $M_{200}$ ). This range is the most massive range sampled by the models and is most appropriate for comparison with Coma and A2029. To account for any systematic difference in the $M_{*}$ and the amplitude, we scale the models to match the observed cluster SMFs using $\chi^{2}$ minimization. For completeness, we show both the scaled and the unscaled model SMFs in Figure 17.

The overall shapes of the model SMFs, regardless of scaling, match the observed SMFs for $\log \left(M_{*} / M_{\odot}\right)>9.5$ remarkably well. Interestingly, both models account for the flat portion of the observed SMFs $\left(9.5<\log \left(M_{*} / M_{\odot}\right)<10.5\right)$. The observed cluster SMFs are incomplete for $\log \left(M_{*} / M_{\odot}\right)<9.5$; thus we cannot test the upturn that appears in both models. At the massive end, $\log \left(M_{*} / M_{\odot}\right)>11.0$, the scaled model SMFs appear to predict too few massive galaxies. This difference is in the same direction that Munari et al. (2016) find between model and observed VDFs for $\log \sigma>2.0$. However, the uncertainty of the model SMFs at $\log \left(M_{*} / M_{\odot}\right)>11.0$ is large (Lim et al. 2017). Nonetheless, the subtle discrepancy could ultimately be a test of models for the formation of the most massive galaxies in clusters.

\subsection{Implications of the Cluster VDFs}

The statistical study of the VDF for quiescent galaxies in clusters complements previous studies of the VDF based on field samples. Galaxy clusters are a useful test bed because the cluster galaxies are essentially at a fixed distance and share the same dense environment. VDFs derived from cluster samples may differ from VDFs based on well controlled field samples as a result of density dependent processes affecting galaxy evolution.

Figure 18 displays the combined cluster VDF for quiescent cluster members with $D_{n} 4000>1.5$ (the sum of the Coma and A2029 VDFs). For comparison, we also plot the VDFs from the SDSS field samples (Sheth et al. 2003; Choi et al. 2007) and from the BOSS field sample (Montero-Dorta et al. 2016). The field VDFs are described by a "modified" Schechter function,

$$
\phi(V) d V=\phi_{0} 10^{\alpha\left(V-V_{*}\right)} \exp \left[-10^{\beta\left(V-V_{*}\right)}\right] \frac{\beta \ln 10}{\Gamma(\alpha / \beta)} d V,
$$

where $V$ is $\log \sigma$. The field VDFs in Figure 18 show the best-fit modified Schechter functions taken from the respective references. We also fit the combined cluster VDF with the modified Schechter functions at $\log \sigma>1.6$. Because the combined cluster VDF is much flatter than the field VDFs at lower $\sigma$, the best-fit parameters differ from the field VDFs. Table 4 lists the best-fit parameters for the cluster VDF and those from the literature.

To compare the overall shape of the VDFs, we scale the field VDFs to match the amplitude of the combined cluster VDF. Unlike the cluster VDF, the field VDFs are normalized by the survey volume. Thus, we scale the amplitude to compare the cluster and field VDFs. Both field VDFs and the cluster VDF are based on $\sigma$ from SDSS/BOSS and comparable Hectospec data. Although the aperture correction methods differ among 


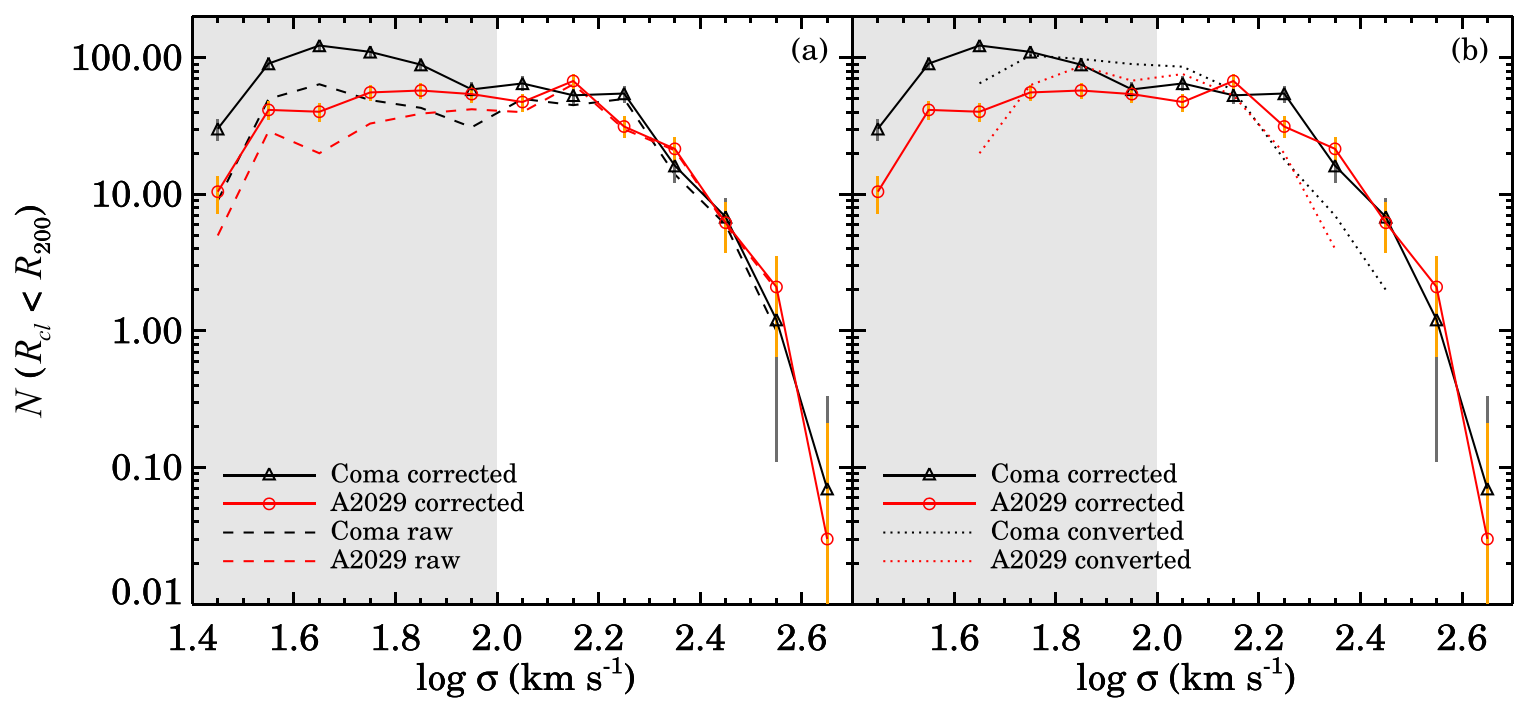

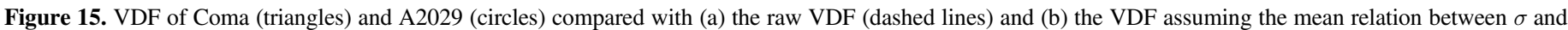
$M_{r}$ (dotted lines) for each cluster. The shaded region indicates the range where incompleteness becomes significant.

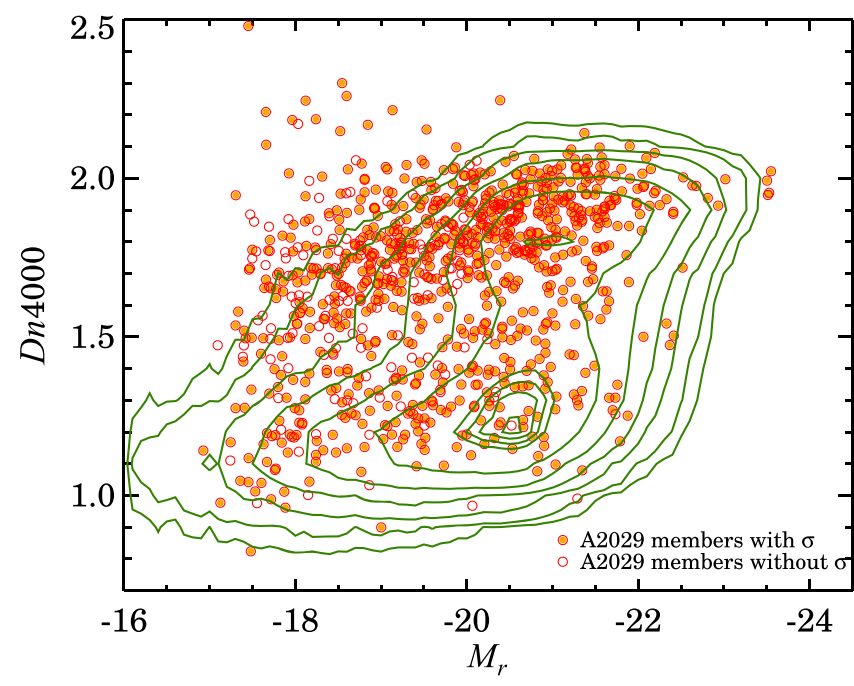

Figure 16. $D_{n} 4000$ vs. $M_{r}$ for A2029 members (symbols) compared with the SDSS field sample (contours). Filled and open circles show A2029 members with $\sigma$ and without $\sigma$, respectively.

these studies, we sample for the $\sigma$ bins for field VDFs without additional calibration to the $\sigma$ aperture we use. The typical aperture correction is only a few percent (Montero-Dorta et al. 2016). Thus, this difference is negligible for the comparison we make here.

Figure 18 underscores the differences between the cluster and field VDFs. Although we scale the field VDFs, the shape difference is significant. At high $\sigma(\log \sigma>2.4)$, the combined cluster VDF substantially exceeds any field VDF. The large $\sigma$ galaxies appearing in clusters are the BCGs, which are rare in field samples.

The discrepancy between cluster and field VDFs is even larger at low $\sigma$; the field VDFs decline rapidly with respect to the cluster VDF. Because the BOSS VDF is limited to $\log \sigma>2.35$, the difference may not be surprising. The SDSS VDFs are substantially shallower than the cluster VDF, although they are complete to $\log \sigma>2.0$.

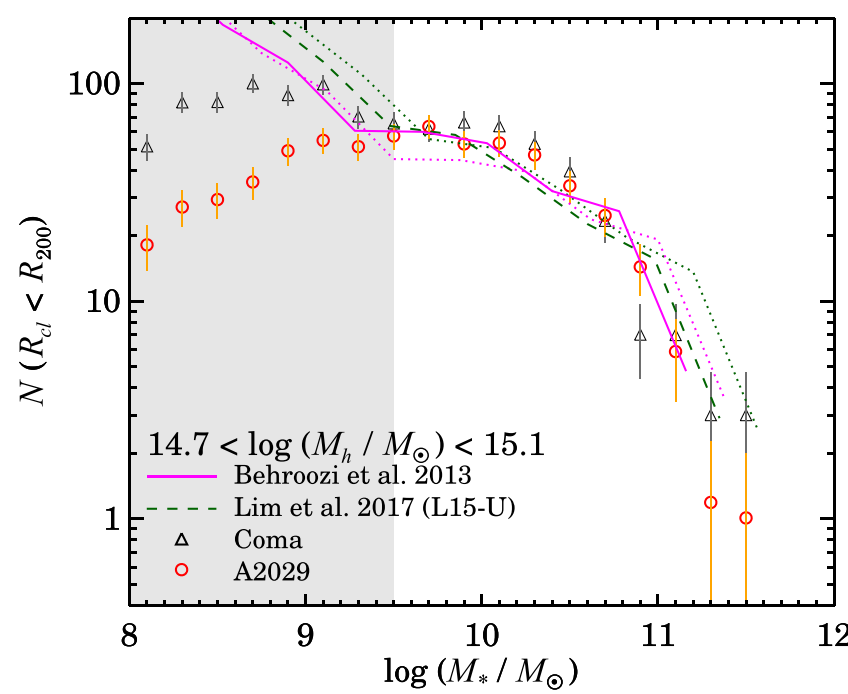

Figure 17. Observed stellar mass functions for Coma (triangles) and A2029 (circles) compared with the model stellar mass functions from Behroozi et al. (2013) (magenta solid lines) and from Lim et al. (2017) (green dashed lines). The model stellar mass functions are scaled to compare the overall shape to the observed stellar mass functions. The magenta dotted lines and green dotted lines show the original unscaled model stellar mass functions from Behroozi et al. (2013) and Lim et al. (2017), respectively.

Different early-type galaxy selection schemes may result in the VDF difference toward low $\sigma$, but not high $\sigma$ (Choi et al. 2007). Our $D_{n} 4000$ selection differs from previous approaches. However, the shape of the combined cluster VDF appears to be insensitive to the specific classification method. For example, the shape of the combined cluster VDF is the same when we measure the cluster VDF based on red-sequence member galaxies rather than galaxies with $D_{n} 4000>1.5$. We note that morphological classification based on the appearance in SDSS images is inadequate for galaxies at the redshift of A2029.

The A2029 and Coma VDFs represent a lower limit to the low $\sigma$ cluster VDF. Missing faint galaxies with $M_{r}<-20$ tend to have generally low $\sigma$. There are also low surface brightness galaxies with $\mu_{r}<24$ mag $\operatorname{arcsec}^{-2}$ missing from our sample. 
Table 4

Velocity Dispersion Function Parameters

\begin{tabular}{|c|c|c|c|c|}
\hline Source & $\sigma$ Range & $\alpha$ & $\beta$ & $\sigma_{*}$ \\
\hline This study & $\log \sigma>1.6$ & $0.00 \pm 0.23$ & $2.47 \pm 0.63$ & $194.85 \pm 26.46$ \\
\hline Sheth et al. (2003) & $\log \sigma>1.95$ & 6.5 & 1.93 & 88.8 \\
\hline Choi et al. (2007) & $\log \sigma>1.84$ & $2.32 \pm 0.10$ & $2.67 \pm 0.07$ & $161.00 \pm 0.05$ \\
\hline Chae (2010) & $\log \sigma>1.9$ & 0.85 & 3.27 & 217.0 \\
\hline Montero-Dorta et al. (2016) & $\log \sigma>2.35$ & $6.75 \pm 0.99$ & $2.37 \pm 0.14$ & $118.86 \pm 12.40$ \\
\hline
\end{tabular}

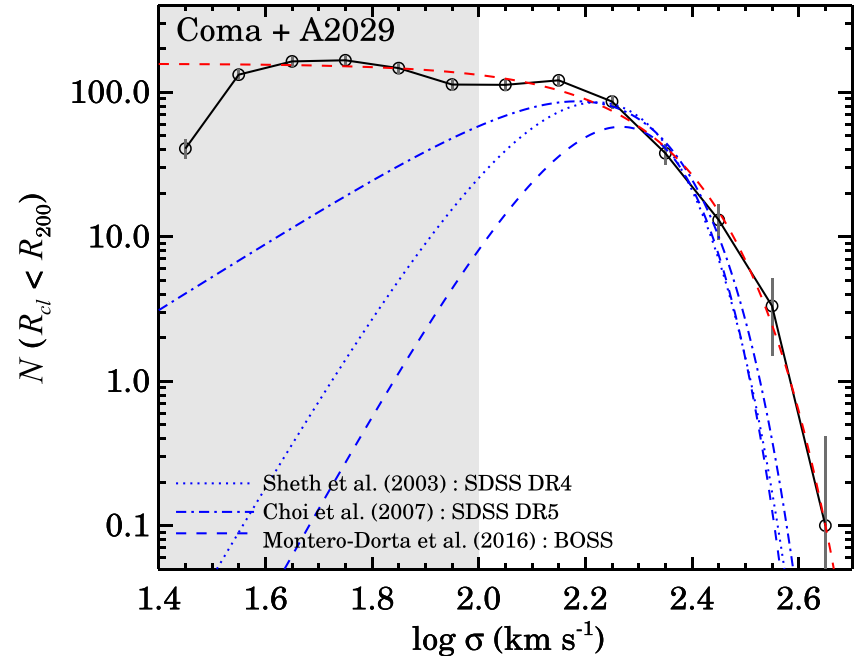

Figure 18. Combined velocity dispersion function (VDF) for A2029 and Coma (solid lines with data points). The red dashed line displays the best-fit modified Schechter function for the combined cluster VDFs. Blue dotted, dotted-dashed, and dashed lines show fitting functions for the velocity dispersion functions derived from SDSS field galaxies (Sheth et al. 2003; Choi et al. 2007) and for BOSS field galaxies (Montero-Dorta et al. 2016), respectively. Note the differences in both the high and low $\sigma$ range.

Thus, the cluster VDF would be steeper at the low $\sigma$ end if it were more complete.

Although the cluster VDFs represent a lower limit at low $\sigma$, the observed cluster VDFs are already much steeper than the field VDFs. The discrepancy between these clusters and the field suggests that corrections made to the field VDFs to account for missing low $\sigma$ galaxies may be inadequate. Choi et al. (2007) measure the SDSS field VDF for galaxies with $M_{r}<-16.8$ in several volume-limited samples. However, a sample that is volume limited is not equivalent to a velocity dispersion-limited sample (Zahid \& Geller 2017). Making the necessary correction for this difference is challenging.

Differences between cluster and field VDFs could be an important window for the related halo mass distribution. The origin of the differences toward high $\sigma$ is an interesting issue because high $\sigma$ BCGs appear predominantly in clusters. In order to interpret the differences between the cluster and field VDFs from a deeper astrophysical perspective, samples that are homogeneous in early-type classification, spectroscopic completeness, and statistics are required both for clusters and the field.

\section{Summary}

We use dense redshift surveys from SDSS and MMT/ Hectospec to identify spectroscopic members of two massive clusters, Coma and A2029. We identify essentially complete samples of $\sim 1000$ spectroscopic members for each cluster based on the caustic technique. To date, only Coma and A2029 have such large samples of spectroscopically identified members. Using the spectroscopic members, we measure the LFs, SMFs, and VDFs for these systems.

The bright end of the cluster LFs is identical to the other cluster LFs derived based on spectroscopic membership. The cluster LFs at the bright end $\left(M_{r}<-18\right)$ are dominated by quiescent (red sequence) galaxies and the slope tends to be flatter than the LF measured over broader luminosity ranges.

The cluster SMFs mimic the cluster LFs. The SMFs are flat to $\log \left(M_{*} / M_{\odot}\right) \sim 9.5$ where the spectroscopic survey is complete. However, the SMFs are somewhat steeper than the LFs over the comparable range. In accounting for missing observations, the translation from luminosity to $M_{*}$ requires the use of conditional probability distribution functions. The resulting observed cluster SMFs are remarkably consistent with simulated SMFs (Behroozi et al. 2013; Lim et al. 2017). A subtle difference at $\log \left(M_{*} / M_{\odot}\right) \sim 11.0$ is interesting because it suggests that the number of massive halos produced in the simulations may be insufficient to match the observations.

For the first time, we derive the cluster VDFs for quiescent cluster members over the broad range $\log \sigma>1.5$. The A2029 VDF and Coma VDF are essentially identical. This similarity suggests that DM subhalo distributions for these two massive clusters are essentially identical.

The cluster VDFs differ from published field VDFs at both high and low $\sigma$. The cluster VDFs exceed the field VDFs at $\sigma \gtrsim 250 \mathrm{~km} \mathrm{~s}^{-1}$ probably reflecting the presence of massive BCGs in the cluster environment. The cluster VDFs also substantially exceed the field VDFs at $\sigma \lesssim 100 \mathrm{~km} \mathrm{~s}^{-1}$ despite the fact that the cluster VDFs represent a lower limit to the count of objects at these dispersions. The differences between cluster and field VDFs are a promising basis for understanding the velocity dispersion and related halo mass distributions in different environments and at various redshifts.

VDFs may be a particularly direct probe of galaxy evolution because several studies suggest that $\sigma$ is a good proxy for the DM subhalo mass (Wake et al. 2012; Bogdán \& Goulding 2015; Zahid et al. 2016). Comparison between the observed VDFs and the simulated quantities calculated directly from hydrodynamic simulations (e.g., Illustris; Vogelsberger et al. 2014 or EAGLE; Schaye et al. 2015) are crucial for a clearer physical understanding of the astrophysical implications of this measure. Like the SMF, the VDF may vary with environment and redshift. Combining simulations that properly mimic the observations with more extensive data could thus provide a new probe of the formation and coevolution of galaxies and their massive halos.

We thank the referee for a clear report that improved the clarity of the paper. We thank Perry Berlind and Michael Calkins for operating Hectospec and Susan Tokarz for helping 
the data reduction. This paper uses data products produced by the OIR Telescope Data Center, supported by the Smithsonian Astrophysical Observatory. We also thank Charles Alcock and Ian Dell'Antonio for insightful discussions. J.S. gratefully acknowledges the support of the CfA Fellowship. The Smithsonian Institution supported the research of M.J.G. and D. F. H.J.Z. is supported by the Clay Postdoctoral Fellowship. A.D. acknowledges support from the the INFN grant InDark, the grant PRIN 2012 Fisica Astroparticellare Teorica of the Italian Ministry of University and Research. K.R. is partially supported by a Cottrell College Science Award from the Research Corporation. This research has made use of NASAs Astrophysics Data System Bibliographic Services. Funding for SDSS-III has been provided by the Alfred P. Sloan Foundation, the Participating Institutions, the National Science Foundation, and the U.S. Department of Energy Office of Science. The SDSS-III web site is http://www. sdss3.org/. SDSS-III is managed by the Astrophysical Research Consortium for the Participating Institutions of the SDSS-III Collaboration including the University of Arizona, the Brazilian Participation Group, Brookhaven National Laboratory, University of Cambridge, Carnegie Mellon University, University of Florida, the French Participation Group, the German Participation Group, Harvard University, the Instituto de Astrofisica de Canarias, the Michigan State/Notre Dame/ JINA Participation Group, Johns Hopkins University, Lawrence Berkeley National Laboratory, Max Planck Institute for Astrophysics, Max Planck Institute for Extraterrestrial Physics, New Mexico State University, New York University, Ohio State University, Pennsylvania State University, University of Portsmouth, Princeton University, the Spanish Participation Group, University of Tokyo, University of Utah, Vanderbilt University, University of Virginia, University of Washington, and Yale University.

\section{References}

Agulli, I., Aguerri, J. A. L., Sánchez-Janssen, R., et al. 2014, MNRAS, 444, L34

Agulli, I., Aguerri, J. A. L., Sánchez-Janssen, R., et al. 2016, MNRAS, 458, 1590

Alam, S., Albareti, F. D., Allende Prieto, C., et al. 2015, ApJS, 219, 12 Andreon, S. 2008, MNRAS, 386, 1045

Andreon, S., \& Cuillandre, J.-C. 2002, ApJ, 569, 144

Arnouts, S., Cristiani, S., Moscardini, L., et al. 1999, MNRAS, 310, 540

Balogh, M. L., Morris, S. L., Yee, H. K. C., Carlberg, R. G., \& Ellingson, E. 1999, ApJ, 527, 54

Barkhouse, W. A., Yee, H. K. C., \& López-Cruz, O. 2007, ApJ, 671, 1471

Behroozi, P. S., Conroy, C., \& Wechsler, R. H. 2010, ApJ, 717, 379

Behroozi, P. S., Wechsler, R. H., \& Conroy, C. 2013, ApJ, 770, 57

Bernardi, M., Meert, A., Sheth, R. K., et al. 2013, MNRAS, 436, 697

Bernardi, M., Shankar, F., Hyde, J. B., et al. 2010, MNRAS, 404, 2087

Blanton, M. R., Dalcanton, J., Eisenstein, D., et al. 2001, AJ, 121, 2358

Bogdán, Á., \& Goulding, A. D. 2015, ApJ, 800, 124

Bruzual, G., \& Charlot, S. 2003, MNRAS, 344, 1000

Calvi, R., Poggianti, B. M., Vulcani, B., \& Fasano, G. 2013, MNRAS, 432,3141

Calzetti, D., Armus, L., Bohlin, R. C., et al. 2000, ApJ, 533, 682

Cappellari, M., \& Emsellem, E. 2004, PASP, 116, 138

Chabrier, G. 2003, PASP, 115, 763

Chae, K.-H. 2010, MNRAS, 402, 2031

Choi, Y.-Y., Park, C., \& Vogeley, M. S. 2007, ApJ, 658, 884

Clarke, T. E., Uson, J. M., Sarazin, C. L., \& Blanton, E. L. 2004, ApJ, 601, 798

Colless, M., \& Dunn, A. M. 1996, ApJ, 458, 435

Conroy, C., Gunn, J. E., \& White, M. 2009, ApJ, 699, 486

Conroy, C., Wechsler, R. H., \& Kravtsov, A. V. 2006, ApJ, 647, 201

Diaferio, A. 1999, MNRAS, 309, 610

Diaferio, A., \& Geller, M. J. 1997, ApJ, 481, 633

Fabricant, D., Chilingarian, I., Hwang, H. S., et al. 2013, PASP, 125, 1362
Fabricant, D., Fata, R., Roll, J., et al. 2005, PASP, 117, 1411 Fabricant, D. G., Kurtz, M. J., Geller, M. J., et al. 2008, PASP, 120, 1222

Fasano, G., Marmo, C., Varela, J., et al. 2006, A\&A, 445, 805

Ferrarese, L., Côté, P., Sánchez-Janssen, R., et al. 2016, ApJ, 824, 10

Fisher, D., Illingworth, G., \& Franx, M. 1995, ApJ, 438, 539

Geller, M. J., Dell'Antonio, I. P., Kurtz, M. J., et al. 2005, ApJL, 635, L125

Geller, M. J., Diaferio, A., \& Kurtz, M. J. 1999, ApJL, 517, L23

Geller, M. J., Hwang, H. S., Dell'Antonio, I. P., et al. 2016, ApJS, 224, 11

Geller, M. J., Hwang, H. S., Fabricant, D. G., et al. 2014, ApJS, 213, 35

Guo, Q., White, S., Li, C., \& Boylan-Kolchin, M. 2010, MNRAS, 404, 1111

Hwang, H. S., Elbaz, D., Lee, J. C., et al. 2010, A\&A, 522, A33

Hwang, H. S., Geller, M. J., Diaferio, A., Rines, K. J., \& Zahid, H. J. 2014 ApJ, 797, 106

Ilbert, O., Arnouts, S., McCracken, H. J., et al. 2006, A\&A, 457, 841

Kauffmann, G., Heckman, T. M., White, S. D. M., et al. 2003, MNRAS, 341,33

Klypin, A., Gottlöber, S., Kravtsov, A. V., \& Khokhlov, A. M. 1999, ApJ, 516, 530

Koleva, M., Prugniel, P., Bouchard, A., \& Wu, Y. 2009, A\&A, 501, 1269

Kravtsov, A. V., Berlind, A. A., Wechsler, R. H., et al. 2004, ApJ, 609, 35

Kurtz, M. J., \& Mink, D. J. 1998, PASP, 110, 934

Laganá, T. F., Zhang, Y.-Y., Reiprich, T. H., \& Schneider, P. 2011, ApJ, 743,13

Lan, T.-W., Ménard, B., \& Mo, H. 2016, MNRAS, 459, 3998

Le Borgne, D., Rocca-Volmerange, B., Prugniel, P., et al. 2004, A\&A, 425,881

Lee, M. G., \& Jang, I. S. 2016, arXiv:1608.05750

Lee, Y., Rey, S.-C., Hilker, M., Sheen, Y.-K., \& Yi, S. K. 2016, ApJ, 822, 92 Li, C., Wang, L., \& Jing, Y. P. 2013, ApJL, 762, L7

Lim, S. H., Mo, H. J., Lan, T.-W., \& Ménard, B. 2017, MNRAS, 464, 3256

Loveday, J., Norberg, P., Baldry, I. K., et al. 2012, MNRAS, 420, 1239

Maraston, C., \& Strömbäck, G. 2011, MNRAS, 418, 2785

McNaught-Roberts, T., Norberg, P., Baugh, C., et al. 2014, MNRAS, 445,2125

Mignoli, M., Cimatti, A., Zamorani, G., et al. 2005, A\&A, 437, 883

Milne, M. L., Pritchet, C. J., Poole, G. B., et al. 2007, AJ, 133, 177

Mobasher, B., Colless, M., Carter, D., et al. 2003, ApJ, 587, 605

Mobasher, B., Dahlen, T., Ferguson, H. C., et al. 2015, ApJ, 808, 101

Montero-Dorta, A. D., Bolton, A. S., \& Shu, Y. 2016, arXiv:1607.06820

More, S., van den Bosch, F. C., Cacciato, M., et al. 2011, MNRAS, 410, 210

Moresco, M., Pozzetti, L., Cimatti, A., et al. 2013, A\&A, 558, A61

Moretti, A., Bettoni, D., Poggianti, B. M., et al. 2015, A\&A, 581, A11

Mortlock, A., Conselice, C. J., Hartley, W. G., et al. 2015, MNRAS, 447, 2

Munari, E., Grillo, C., De Lucia, G., et al. 2016, ApJL, 827, L5

Okabe, N., Futamase, T., Kajisawa, M., \& Kuroshima, R. 2014, ApJ, 784, 90

Park, C., \& Hwang, H. S. 2009, ApJ, 699, 1595

Paterno-Mahler, R., Blanton, E. L., Randall, S. W., \& Clarke, T. E. 2013, ApJ, 773, 114

Rines, K., \& Geller, M. J. 2008, AJ, 135, 1837

Rines, K., Geller, M. J., Diaferio, A., \& Kurtz, M. J. 2013, ApJ, 767, 15

Rines, K., Geller, M. J., Kurtz, M. J., \& Diaferio, A. 2003, AJ, 126, 2152

Rines, K. J., Geller, M. J., Diaferio, A., \& Hwang, H. S. 2016, ApJ, 819, 63

Sánchez-Blázquez, P., Peletier, R. F., Jiménez-Vicente, J., et al. 2006, MNRAS, 371, 703

Schaye, J., Crain, R. A., Bower, R. G., et al. 2015, MNRAS, 446, 521

Schechter, P. 1976, ApJ, 203, 297

Serra, A. L., \& Diaferio, A. 2013, ApJ, 768, 116

Serra, A. L., Diaferio, A., Murante, G., \& Borgani, S. 2011, MNRAS, 412, 800

Shen, S.-Y., Argudo-Fernández, M., Chen, L., et al. 2016, RAA, 16, 007

Sheth, R. K., Bernardi, M., Schechter, P. L., et al. 2003, ApJ, 594, 225

Sohn, J., Hwang, H. S., Geller, M. J., et al. 2015, JKAS, 48, 381

Springel, V., Wang, J., Vogelsberger, M., et al. 2008, MNRAS, 391, 1685

Strauss, M. A., Weinberg, D. H., Lupton, R. H., et al. 2002, AJ, 124, 1810

Swindle, R., Gal, R. R., La Barbera, F., \& de Carvalho, R. R. 2011, AJ, 142,118

Thomas, D., Steele, O., Maraston, C., et al. 2013, MNRAS, 431, 1383

Tinker, J. L., Brownstein, J. R., Guo, H., et al. 2016, arXiv:1607.04678

Tyler, K. D., Rieke, G. H., \& Bai, L. 2013, ApJ, 773, 86

Uson, J. M., Boughn, S. P., \& Kuhn, J. R. 1991, ApJ, 369, 46

Vale, A., \& Ostriker, J. P. 2004, MNRAS, 353, 189

Vale, A., \& Ostriker, J. P. 2006, MNRAS, 371, 1173

van Uitert, E., Hoekstra, H., Franx, M., et al. 2013, A\&A, 549, A7

Vergani, D., Scodeggio, M., Pozzetti, L., et al. 2008, A\&A, 487, 89 
Vogelsberger, M., Genel, S., Springel, V., et al. 2014, MNRAS, 444, 1518

Vulcani, B., Poggianti, B. M., Aragón-Salamanca, A., et al. 2011, MNRAS, 412, 246

Vulcani, B., Poggianti, B. M., Oemler, A., et al. 2013, A\&A, 550, A58

Wake, D. A., Franx, M., \& van Dokkum, P. G. 2012, arXiv:1201.1913

Walker, S. A., Fabian, A. C., Sanders, J. S., George, M. R., \& Tawara, Y. 2012 MNRAS, 422, 3503
White, S. D. M., Clowe, D. I., Simard, L., et al. 2005, A\&A, 444, 365

Woods, D. F., Geller, M. J., Kurtz, M. J., et al. 2010, AJ, 139, 1857

Yang, X., Mo, H. J., \& van den Bosch, F. C. 2008, ApJ, 676, 248

Zahid, H. J., Dima, G. I., Kudritzki, R.-P., et al. 2014, ApJ, 791, 130

Zahid, H. J., \& Geller, M. J. 2017, arXiv:1701.01350

Zahid, H. J., Geller, M. J., Fabricant, D. G., \& Hwang, H. S. 2016, ApJ, 832,203 